\title{
A numerical study into the morphology and formation mechanisms of threedimensional particle structures in vibrated cylindrical cavities with various heating conditions
}

\author{
Marcello Lappa \\ Department of Mechanical and Aerospace Engineering, University of Strathclyde \\ James Weir Building, 75 Montrose Street, Glasgow, G1 1XJ, UK \\ email: marcello.lappa@strath.ac.uk - marlappa@unina.it
}

\begin{abstract}
The present analysis extends the author's earlier work (Lappa, Phys. Fluids, 26, 093301, 2014) on the properties of patterns formed by the spontaneous accumulation and ordering of solid particles in certain types of flow. It is shown that under certain conditions, when subjected to vibrations to induce natural flow, non-isothermal fluids with dispersed solid particles are characterized by intervals of solid-pattern-forming behaviour due to particle rearrangements preceded by intervals in which no recognizable structures of solid matter can be detected. The dynamics of these systems are highly nonlinear in nature. Because this new family of particle attractors is known to exhibit strong sensitivity to the "symmetry properties" of the considered vibrated system and related geometrical constraints, the present study attempts to clarify the related dynamics in a geometry with curved walls (cylindrical enclosure). In particular, by assuming vibrations always directed perpendicularly to the imposed temperature gradient, we show that the morphology, spatial extension (percentage of physical volume occupied), "separation" (spatial distance) and mechanisms responsible for the formation of the resulting particle structures change significantly according to whether the temperature gradient is parallel or perpendicular to the symmetry axis of the cylinder. This indicates that the "physics" is not invariant with respect to $90^{\circ}$ rotations in space of the specific forcing considered (direction of the imposed temperature gradient and associated perpendicular vibrations). Additional insights into the problem are obtained by assessing separately the influence played by the time-averaged (mean) and oscillatory effects. According to the numerical results, the intriguing diversity of particle agglomerates results from the different role/importance played by (curved or straight) boundaries in constraining particles and, from the different structure and topology of the resulting macroscopic (large-scale) thermovibrational flow oscillating in time at the same acceleration frequency of the imposed vibrations.
\end{abstract}

Keywords: Cylindrical cavity, Thermovibrational flow, Particle clustering phenomena. 


\section{Introduction}

Fluids with dispersed solid particles have been widely used in industry. These applications span such diverse fields as cooling systems for nuclear reactors, heat exchangers, solar energy collectors, electronic industry and a variety of modern processes for the production of new materials with improved physical and chemical properties (e.g., structural materials for marine and aerospace engineering, many types of composites, electrical conductors, and magnetic substances). Notably, the properties of these products are linked to their microscopic structure (their electrical and/or mechanical properties can be ascribed to characteristics such as high disorder, caging, and/or particle clustering on multiple length scales), which makes the study of the solid-liquid pattern formation a topic of great interest to materials engineers (see, e.g., Segurado et al. [1]).

In spite of their great potentials, however, these special fluids are still in an early stage of exploitation. Indeed, from a theoretical viewpoint, such solid-liquid mixtures still represent a rather complex problem because of the fact that it appears very difficult, if not to say practically impossible, to formulate any theory that can predict "a priori" their behaviour.

Among the phenomena to be taken into account for a possible prediction of the final distribution of particles in the liquid matrix, one should mention: sedimentation (in the Earth's gravitational field the usual differences in density cause rapid spatial separation of the solid phase through sedimentation or flotation), the application of other body forces (e.g., electric and/or magnetic fields) and inertial clustering (the process of spontaneous grain aggregation determined by particle specific inertia and viscous drag in the presence of fluid convection).

In the present work, given the recent resurgence of theoretical studies exploring the consequences of particle clustering mechanisms [2-11] and in view of the generality of these structure-forming processes, we expressly concentrate on situations where particle inertia acts as the main patternforming driver. In particular, we consider particle aggregation and ordering mechanisms operating under the effect of "alternating" flows induced by "vibrations" (i.e. body accelerations varying sinusoidally in time). The specificity of the considered situation lies in the nature of the driving force, which has zero-time averaged value and which, accordingly, makes linear effects negligible; thereby only nonlinear effects are expected to be significant (even if they are small compared to the instantaneous linear effects). The reason of such a choice also resides in the fact that, like magnetic fields, vibrations allow contactless control of the flow and dispersed particles, but, unlike magnetic fields, they may be regarded as a new technique that can be used more universally, because its application is not limited to electrically conductive melts and particles.

Since the author's earlier analysis [12] was entirely concentrated on a geometrical configuration with straight walls (a cubical cavity) and a fixed direction of the temperature gradient, here we allow the problem to span two additional degrees of freedom, namely the presence of curved sidewalls (cylindrical configuration) and the relative direction of the imposed temperature gradient and vibrations with respect to such sidewalls. 
Natural convection induced by steady (terrestrial) gravity in fluid-filled cylindrical enclosures has received enormous attention over the past several years due to its wide applications in engineering and technology [13-22]. For similar studies considering convective effects induced by time-varying accelerations rather than steady gravity, the reader may consider [23-31].

For what concerns the dynamics of solid particles transported by natural flows in such cylindrical configurations, available efforts seem to be very rare and sparse. Most of analyses have concentrated on the case of particle clustering supported by Marangoni convection ([32] and references therein, see also [33, 34]), or Rayleigh-Bénard flow [35], which, however, pertain to a different category of phenomena.

As outlined above, in this analysis, neither a steady external force field nor surface-tension-driven effects are considered; rather the dynamics are entirely produced by the "interplay" between an acceleration field varying sinusoidally in time, the resulting thermovibrational flow and the response of particles (depending on their specific mass and size).

\section{Mathematical formulation}

\section{A. Vibrations}

Perturbations induced in a fluid by a sinusoidal displacement of a fluid system along a given direction ( $\underline{\hat{n}}$ is the related unit vector) $\underline{s}(t)=b \sin (\omega t) \underline{\hat{n}}$ where $b$ is the amplitude and $\omega=2 \pi f$ ( $\mathrm{f}$ is the frequency), induce an acceleration $\underline{g}(t)=g_{\omega} \sin (\omega \mathrm{t}) \underline{\hat{n}}$ where $g_{\omega}=\mathrm{b} \omega^{2}$ [36]. Such inertial perturbations are known to affect "inhomogeineities" present in the considered fluid system (see, e.g. [37]). These inhomogeineities may be due, on the one hand, to density variations induced in the fluid by temperature gradients (which lead to the so-called thermovibrational flow, [38-40]) and, on the other hand, (see, e.g., [41]) to the granular structure of the considered two-phase system (an assembly of hard spheres dispersed in the liquid in the present case). The related principles and governing equations will be illustrated in Sects. IIC and IID, respectively.

\section{B. The System}

Insights are sought from consideration of an archetypal setting corresponding to a differentially heated cylindrical cavity. In particular, a cylindrical enclosure with height/diameter ratio $\mathrm{A}=\mathrm{L} / \mathrm{D}=0.75$ filled with $\mathrm{NaNO}_{3}$ (sodium nitrate with $\mathrm{Pr}=8$ ) is considered. Following earlier computational studies reported in this area for the case of steady gravity (see the introduction for existing studies in the literature), three main configurations are defined in the present work for what concerns thermal boundary conditions: a) Differentially heated cylindrical enclosure with adiabatic conditions on the lateral wall, b) Cylindrical enclosure limited axially by insulated solid walls with a temperature on the lateral wall varying as a sinusoidal function of the azimuthal angle, c) intermediate configuration with temperature varying along both the axial and the azimuthal directions (Fig. 1) 
As the flow of thermovibrational nature induced by vibrations is known to be very sensitive to the relative angle between the imposed temperature gradient and the direction of vibrations [42], with the emerging convection being very weak or absent when such directions are parallel (degenerate situation), here, in particular, without loss of generality, we concentrate expressly on the case of imposed periodic accelerations perpendicular to the imposed temperature difference $\Delta \mathrm{T}$ (Fig. 1).

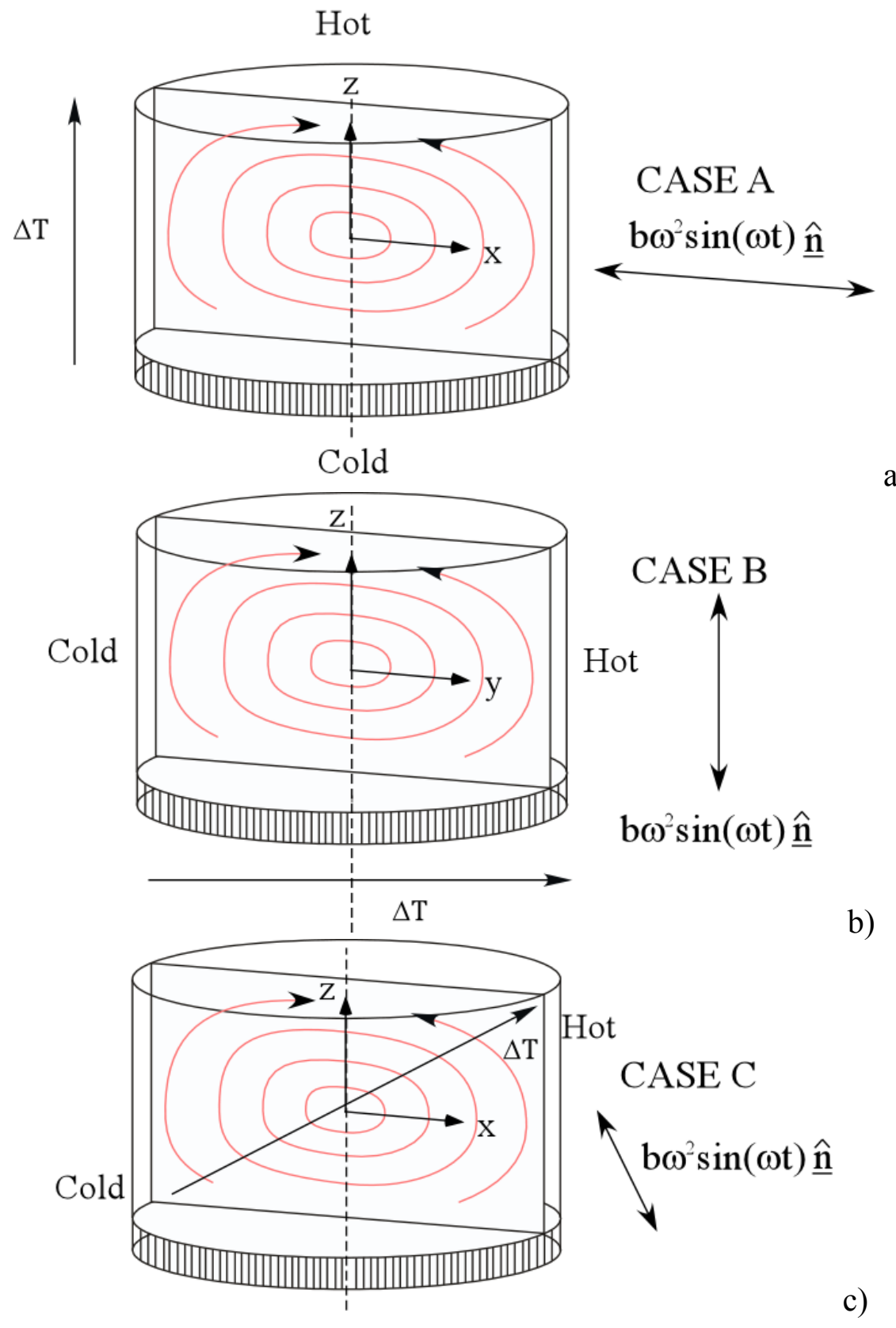

Figure 1: Sketch of configuration under investigation: a) Cylinder with differentially heated ends and adiabatic sidewall subjected to vibrations perpendicular to its axis, b) Cylinder with insulated ends and nonisothermal sidewall (temperature varying as a sinusoidal function of the azimuthal angle) subjected to vibrations parallel to its axis, c) Cylinder with temperature varying on the boundary along both the axial and the azimuthal direction and vibrations perpendicular to the imposed temperature gradient. 


\section{Nondimensional balance equations}

Variables are non-dimensionalized using conventional scalings: the co-ordinates by the axial system extension (L) and the velocity $\underline{\mathrm{V}}$ by the energy diffusion velocity $\mathrm{V}_{\alpha}=\alpha / \mathrm{L}$ where $\alpha$ is the fluid thermal diffusivity; time and pressure (p) by, respectively, $L^{2} / \alpha$ and $\rho \alpha^{2} / L^{2}$, where $\rho$ is the fluid density. Moreover, the temperature is measured with respect to its initial value $\overline{\mathrm{T}}_{\mathrm{m}}$ and is made nondimensional as $\mathrm{T}=\left(\overline{\mathrm{T}}-\overline{\mathrm{T}}_{\mathrm{m}}\right) / \Delta \mathrm{T}$.

In such a framework and using the Boussinesq approximation to account for the presence of body accelerations as defined in Sect. IIA, the continuity and momentum equations simply read:

$\underline{\nabla} \cdot \underline{\mathrm{V}}=0$

$\frac{\partial \underline{V}}{\partial}=-\underline{\nabla} p-\underline{\nabla} \cdot[\underline{V} \underline{V}]+\operatorname{Pr} \nabla^{2} \underline{V}+\operatorname{Pr} \frac{b \omega^{2} \beta_{T} \Delta T L^{3}}{v \alpha} T \sin \left(\frac{L^{2} \omega}{\alpha} t\right) \underline{\hat{n}}$

where $\frac{b \omega^{2} \beta_{T} \Delta T L^{3}}{v \alpha}=R a_{\omega}$ can be regarded as a variant of the classical Rayleigh number with the steady acceleration being replaced by the amplitude of the considered periodic acceleration $\left(\beta_{\mathrm{T}}\right.$ is the thermal expansion coefficient). The problem, therefore, is reduced to four independent nondimensional parameters only, where the first is the well-known Prandtl number $(\mathrm{Pr}=v / \alpha$ where $v$ is the fluid kinematic viscosity) and the others are the nondimensional frequency $(\varpi)$, the nondimensional acceleration amplitude $(\gamma)$ and buoyancy factor $(\beta)$ defined as:

$\varpi=\frac{\omega L^{2}}{\alpha}$

$\gamma=\frac{b \omega^{2} L^{3}}{\alpha^{2}}$

$\beta=\left(\beta_{T} \Delta T\right)$

Accordingly, the momentum equation can be recast in condensed form as:

$\frac{\partial \underline{V}}{\partial t}=-\underline{\nabla} p-\underline{\nabla} \cdot[\underline{V} \underline{V}]+\operatorname{Pr} \nabla^{2} \underline{V}+\gamma \beta T \sin (\varpi t) \underline{\hat{n}}$

where, obviously $\operatorname{Ra}_{\omega}=\gamma \beta / \operatorname{Pr}$.

This equation must be supplemented with the energy balance equation that with the considered reference units in nondimensional form reads:

$\frac{\partial T}{\partial t}+\underline{\nabla} \cdot[\underline{V} T]=\nabla^{2} T$ 
For $\mathrm{t}=0$, initial conditions corresponding to liquid at rest and thermally diffusive conditions are assumed. For $\mathrm{t}>0$, the boundary conditions at the solid walls simply reflect the well-known no-slip and impermeability properties of solid boundaries $(\underline{\mathrm{V}}=0)$. We do not report them here explicitly for the sake of brevity (and given their extreme simplicity). For problem closure, such conditions, however, have to be supplemented with those for the energy equation, which change according to whether the configuration of Fig. 1a, 1b or 1c is considered. In the first case, the top and bottom $(\mathrm{z}= \pm 0.5)$ walls of the domain are assumed here to be at uniform and constant (nondimensional) temperatures:

$$
\begin{array}{ll}
\mathrm{T}_{0}=1 / 2 & \text { at }(\mathrm{z}=-0.5) \\
\mathrm{T}_{1}=-1 / 2 & \text { at }(\mathrm{z}=0.5)
\end{array}
$$

while the lateral boundary is adiabatic, i.e.

$\partial \mathrm{T} / \partial \mathrm{r}=0$ at $\mathrm{r}=1 / 2 \mathrm{~A}, 0 \leq \varphi \leq 2 \pi,-0.5<\mathrm{z}<0.5$

For the second configuration, such conditions have to be replaced by

$\partial \mathrm{T} / \partial \mathrm{z}=0$ at $(\mathrm{z}= \pm 0.5)$

$\mathrm{T}=1 / 2 \sin (\varphi)$ at $\mathrm{r}=1 / 2 \mathrm{~A}, 0 \leq \varphi \leq 2 \pi,-0.5<\mathrm{z}<0.5$

For the third case, they read

$\mathrm{T}=-1 / 2[1 / 2 \sin (\varphi)+\mathrm{z}]$ at $\mathrm{r}=1 / 2 \mathrm{~A}, 0 \leq \varphi \leq 2 \pi,-0.5 \leq \mathrm{z} \leq 0.5$

$\mathrm{T}=-1 / 2[1 / 2 \sin (\varphi)+\mathrm{z}]$ at $0 \leq \mathrm{r} \leq 1 / 2 \mathrm{~A}, 0 \leq \varphi \leq 2 \pi$ at $(\mathrm{z}= \pm 0.5)$

\section{The Dispersed Phase}

The dynamics of particles are treated using the approach originally introduced by Maxey and Riley [43]). Such approach relies on the assumption that the particle velocity can be decomposed into two different contributions, i.e. $\underline{\mathrm{V}}_{\text {part }}(\mathrm{r}, \mathrm{t})=\underline{\mathrm{V}}(\mathrm{r}, \mathrm{t})+\mathrm{v}_{1}(\mathrm{r}, \mathrm{t})$, where $\underline{\mathrm{V}}$ is the velocity of the undisturbed flow at the same spatial position occupied by the considered particle (which would result if the boundary conditions at the particle surface were not applied), while $\mathrm{v}_{1}$ has to be regarded as the "perturbative" component created by the fluid-particle interaction.

From physical arguments, it is known that the fluid force on the particle depends on the local fluid inertia (proportional to the local material derivative of $\underline{\mathrm{V}}$ ) and also to the local stress created by the presence of the particle. In particular, the relaxational part of the particle inertia is strictly related to 
its mass resistance (preventing it from following instantaneously the velocity of the surrounding fluid), whereas the fluid drag tends to damp the particle velocity towards the local fluid velocity (within an inertial time $\tau$ that scales with the particle radius $\widetilde{R}$ as $\tau=2 \widetilde{R}^{2} / 9 v$ ) [44].

These effects can be properly modeled by considering relevant viscous and inertial terms in the aforementioned Lagrangian equations used to track the evolution of the particle in space and time.

Despite this obvious way of thinking, however, some additional simplifying assumptions have to be invoked. These additional conditions concern the reciprocal influence of particle motion on the large-scale flow and the particle-particle interplay ([44-46]). Following a common practice in the related literature ([32]), here the so-called dilute particle model is used, i.e. it is assumed that while particles are transported by the large-scale flow, the influence they exert on such a flow and among them is negligible, in other words, the perturbative flow $\mathrm{v}_{1}$ has a negligible effect on the unperturbed field (in the presence of vibrations, as shown by several authors, this assumption is reliable if the concentration of the dispersed phase in the flow is small, i.e. if the distance of a particle from others is much larger than their diameter; see, e.g. [2, 47-48]; the interested reader is referred, in particular, to Figure 4 in [2]).

Within such a framework, the resulting particle transport equation reads:

$$
\begin{aligned}
& \frac{d u_{\text {partcl }}}{d t}=\frac{1}{\xi+1 / 2}\left[-\frac{\operatorname{Pr}}{S t}\left(u_{\text {partcl }}-u\right)+\frac{3}{2} \frac{\partial u}{\partial t}+\frac{3}{2}(\underline{V} \cdot \underline{\nabla} \underline{V})_{u}\right]+\frac{\xi-1}{\xi+1 / 2} \gamma \sin (\varpi t) \sin (\phi) \\
& \frac{d v_{\text {partcl }}}{d t}=\frac{1}{\xi+1 / 2}\left[-\frac{\operatorname{Pr}}{S t}\left(v_{\text {partcl }}-v\right)+\frac{3}{2} \frac{\partial v}{\partial t}+\frac{3}{2}(\underline{V} \cdot \underline{\nabla} \underline{V})_{v}\right]+\frac{\xi-1}{\xi+1 / 2} \gamma \sin (\varpi t) \cos (\varphi) \cos (\phi) \\
& \frac{d w_{\text {partcl }}}{d t}=\frac{1}{\xi+1 / 2}\left[-\frac{\operatorname{Pr}}{S t}\left(w_{\text {partcl }}-w\right)+\frac{3}{2} \frac{\partial w}{\partial t}+\frac{3}{2}(\underline{V} \cdot \underline{\nabla} \underline{V})_{w}\right]+\frac{\xi-1}{\xi+1 / 2} \gamma \sin (\varpi t) \sin (\varphi) \cos (\phi)
\end{aligned}
$$

where $\phi$ is the angle between the direction of the imposed vibrations and the xy plane, $\xi$ is the ratio of the particle to the fluid density and $\left(\mathrm{u}_{\text {part }}, \mathrm{v}_{\text {part }}, \mathrm{w}_{\text {part }}\right)$ and $(\mathrm{u}, \mathrm{v}, \mathrm{w})$ are the particle and fluid (axial, radial and azimuthal) velocity components, respectively. Moreover

$$
\begin{aligned}
& (\underline{V} \cdot \underline{\nabla} \underline{V})_{u}=\left(u \frac{\partial u}{\partial z}+v \frac{\partial u}{\partial r}+\frac{w}{r} \frac{\partial u}{\partial \varphi}\right) \\
& (\underline{V} \cdot \underline{\nabla} \underline{V})_{v}=\left(u \frac{\partial}{\partial z}+v \frac{\partial v}{\partial r}+\frac{w}{r} \frac{\partial v}{\partial \varphi}-\frac{w^{2}}{r}\right) \\
& (\underline{V} \cdot \underline{\nabla} \underline{V})_{w}=\left(u \frac{\partial w}{\partial z}+v \frac{\partial w}{\partial r}+\frac{v w}{r}+\frac{w}{r} \frac{\partial w}{\partial \varphi}\right)
\end{aligned}
$$


As discussed, e.g., in [41], the underlying assumptions on which this approach is based are a perfectly spherical shape of the solid particles and a very small value $(<<1)$ of the particle to fluid system characteristic size ratio $\widetilde{R} / L$ (where $\widetilde{R}$ is the solid particle radius). This implies $\mathrm{St}=\tau v / \mathrm{L}^{2}$ $<<1$ where $\tau$ is the aforementioned particle relaxation time and $\mathrm{St}$ is the well-known Stokes number. Although equation (9) properly accounts for the motion of solid particles in the liquid phase, it is clear, however, that particles moving close to the solid boundaries require a special treatment (given the no-slip properties of such surfaces acting as sinks of momentum and, therefore, as a potential particle-entrapping loci). Following earlier studies, in particular, we assume particles to interact in a non-elastic fashion with walls [32,33].

\section{E. Numerical Method and Code Validation for Buoyancy Flow}

Balance equations (1-5) have been solved numerically by a time-explicit finite-difference method (primitive-variable method) based on a cylindrical mesh and a staggered collocation of fluiddynamic variables. Forward differences in time and central-differencing schemes in space (second order accurate) have been used to discretize the energy and momentum governing equations. The related solution strategy is not discussed here, the interested reader being referred to various books in the literature for an exhaustive treatment, see, e.g. [49] (here, we just limit ourselves to recalling that such an approach is based on the well-known pressure-velocity coupling strategy based on the application of the momentum equation to derive an equation for pressure via the time-discretized continuity equation). For the specific case of convection induced by buoyancy the present code was validated through comparison with the classical 3D solutions reported by Bontoux et al. [16], see Fig. 2.

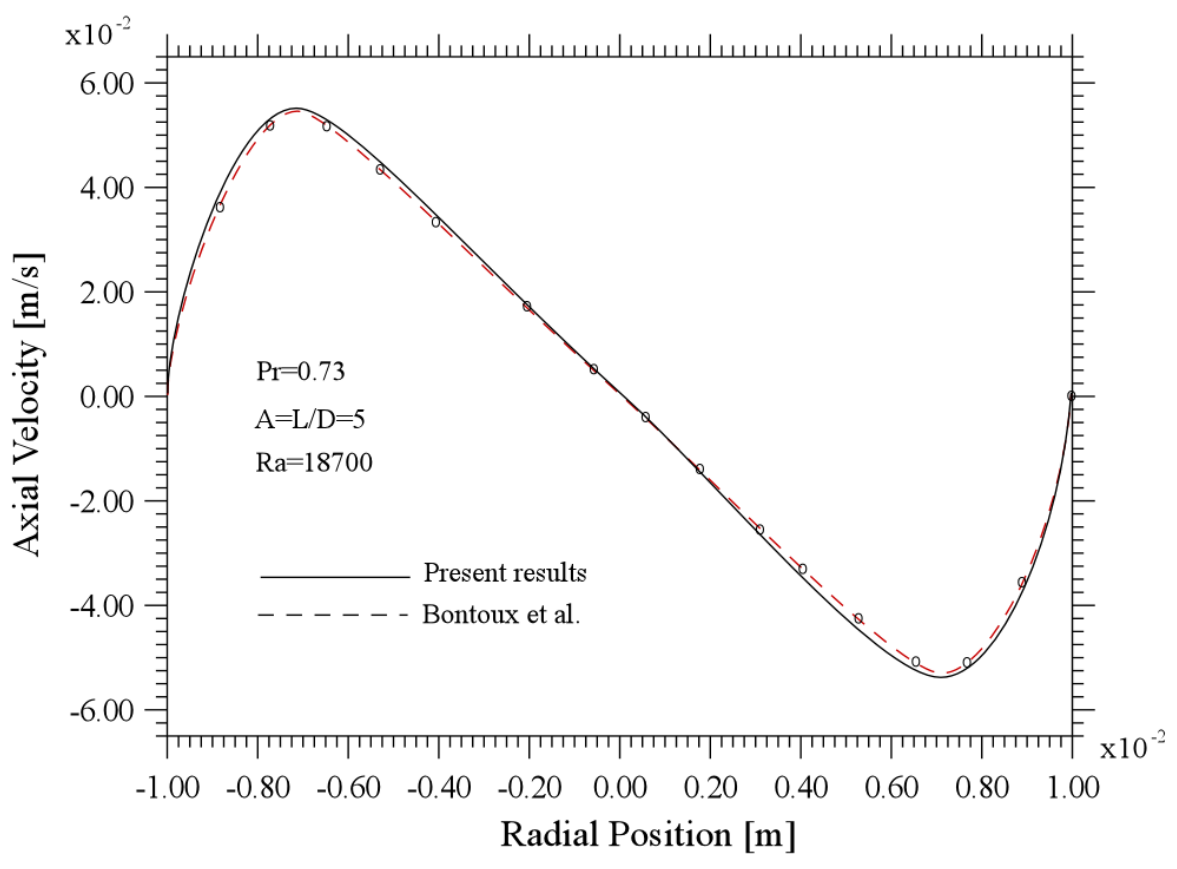

Figure 2: Comparison with the results reported by Bontoux et al. [16]: differentially heated horizontal cylinder with $\mathrm{L}=10^{-1} \mathrm{~m}, \mathrm{~A}=5, \mathrm{Pr}=0.73, \mathrm{Ra}=1.87 \times 10^{4}\left(\mathrm{~N}_{\mathrm{Z}} \times \mathrm{N}_{\mathrm{r}} \mathrm{xN} \mathrm{N}_{\varphi}\right.$ mesh $\left.65 \times 16 \times 32\right)$. 


\subsection{Case of interest}

The properties of the considered solid-liquid system are summarized in Table I.

TABLE I: Liquid-solid system properties

\begin{tabular}{ll}
\hline Fluid $\left(\mathrm{NaNO}_{3}\right)$ density $\rho,\left[\mathrm{kg} \mathrm{m}^{-3}\right]$ & 1904 \\
Kinematic viscosity $v,\left[\mathrm{~m}^{2} \mathrm{~s}^{-1}\right]$ & $1.27 \times 10^{-6}$ \\
Thermal diffusivity $\alpha,\left[\mathrm{m}^{2} \mathrm{~s}^{-1}\right]$ & $1.58 \times 10^{-7}$ \\
Thermal expansion coefficient $\beta_{\mathrm{T}},\left[\mathrm{K}^{-1}\right]$ & $1.25 \times 10^{-3}$ \\
Particle/liquid density ratio $\xi[-]$ & 1.85 \\
\hline \hline
\end{tabular}

Assuming as axial extension of the cylindrical enclosure $\mathrm{L}=2 \times 10^{-2} \mathrm{~m}$, the following values of characteristic nondimensional parameters are considered: $\varpi=10^{3}, \gamma=1.5 \times 10^{6}$ which correspond to $\operatorname{Ra}_{\omega}=1.58 \times 10^{4}$. This is also equivalent to considering a value of the so-called Gershuni Number:

$G s=\frac{\left(b \omega \beta_{T} \Delta T L\right)^{2}}{2 v \alpha}=\frac{\left(\beta_{T} \Delta T L\right)^{2}}{2 v \alpha}\left(\frac{g_{\omega}}{\omega}\right)^{2}=\frac{\varpi^{2} \Lambda^{2}}{2 \operatorname{Pr}}=\frac{1}{2 \operatorname{Pr}}\left(\frac{\beta \gamma}{\varpi}\right)^{2}=10^{3}$

Such a parameter (which, in general, accounts for the intensity of time-averaged convection resulting from the application of high-frequency vibrations, see, e.g., [42]), is fixed to Gs $=10^{3}$, so as to guarantee that time-averaged convective effects of thermovibrational nature are very small with respect to the oscillatory component of fluid velocity. This also guarantees a negligible departure of the time-averaged temperature field from the purely diffusive conditions (i.e. the distribution of temperature inside the enclosure can be assumed to be almost linear).

The density of the particles is also considered fixed: $\xi=1.85$, such a value corresponding to typical tracers used in experiments with $\mathrm{NaNO}_{3}$ (Melnikov et al. [4]). Their characteristic size gives $\eta=(\xi$ 1) $\mathrm{St} / \mathrm{Pr}=10^{-4}$, this value of $\eta$ being a good compromise between two opposite needs (as further explained in the following). As shown by Lappa [12] (to which the reader is referred for an exhaustive treatment of such aspects), the formation of recognizable particle structures in the presence of thermovibrational flow takes a time that decreases as the particle size is increased; particle accumulation, however, is found not to occur when the particle size exceeds a given threshold. Accordingly, the specific value $\eta=10^{-4}$ has been selected in the present work with the two-fold purpose to shorten the otherwise computationally prohibitive time required for the formation of well-defined particle patterns on the one hand, and not to exceed the abovementioned limit in terms of particle size (beyond which such patterns are no longer formed), on the other hand. 


\section{F. Grid Refinement Study and Code Validation for Thermovibrational Convection}

The ability of the present code to correctly capture the particle dynamics in flows of "oscillatory nature" in cylindrical geometries was already assessed to a certain extent in earlier studies. As an example this was verified for the case of a "travelling wave" in [33] (where the results provided by the present code were quantitatively compared with those by Melnikov et al. [4]) and in Lappa [34] for the case of a "standing wave" (where the present code was used to reproduce the dynamics presented by Schwabe and Mizev [10]). The limits of applicability of the considered multiphase approach in capturing particle dynamics induced by high-frequency vibrations were further assessed by [41] (all these studies considered the same value of the Prandtl number assumed here, namely $\operatorname{Pr}=8)$.

As usual, in the present work, the convergence behavior of the code in terms of its ability to produce a mesh independent solution (both in terms of fluid velocity field and resulting particle structures) has been assessed via an "a priori” grid tailoring process.

In particular, such an assessment has been conducted assuming as "sensitive" parameters (to check grid convergence) the maximum (asymptotic) value attained by each of the velocity components $u$, $\mathrm{v}, \mathrm{w}$. Owing to the nature of the specific problem under investigation (able to produce a "steady mean flow" field as well as well-defined particle structures with time-independent "properties"), such a study has also taken into account time-averaged quantities and "measurable" characteristics of the emerging particulate structures. The time-averaged velocity components have been computed, respectively, as

$$
\langle u\rangle=\frac{\varpi}{2 \pi} \int_{0}^{2 \pi / \pi} u(t) d t \quad\langle v\rangle=\frac{\varpi}{2 \pi} \int_{0}^{2 \pi / \pi} v(t) d t \quad\langle w\rangle=\frac{\varpi}{2 \pi} \int_{0}^{2 \pi / \pi} w(t) d t
$$

with the velocity components being provided by the solution of the set of equations presented in Sect. IIC

For code validation purposes, these quantities (and related patterns in space) have been also determined resorting to an independent code based on the solution of the so-called Gershuni's system of equation. These alternate sets of equations, based on the introduction of a "potential flow" variable, can be used to determine directly the time-averaged flow with no need to solve the complete set of Navier-Stokes equations (these equations, traditionally used in the literature for validation purposes in the case of flows with thermovibrational nature, are not described here due to page limits, the reader being referred, e.g. to [38] for additional details).

As a relevant and representative case for such an extensive assessment, in particular, we focused on the case corresponding to the conditions shown in Fig. 1b, for which the emerging particle structures have a relatively simple shape that allows their typical "size" to be measured precisely (namely, the "lengths" e1 and e2, representing the axes of the elliptical cross section given by the intersection of the emerging particle structures with the center plane yz, see Fig. 3) 


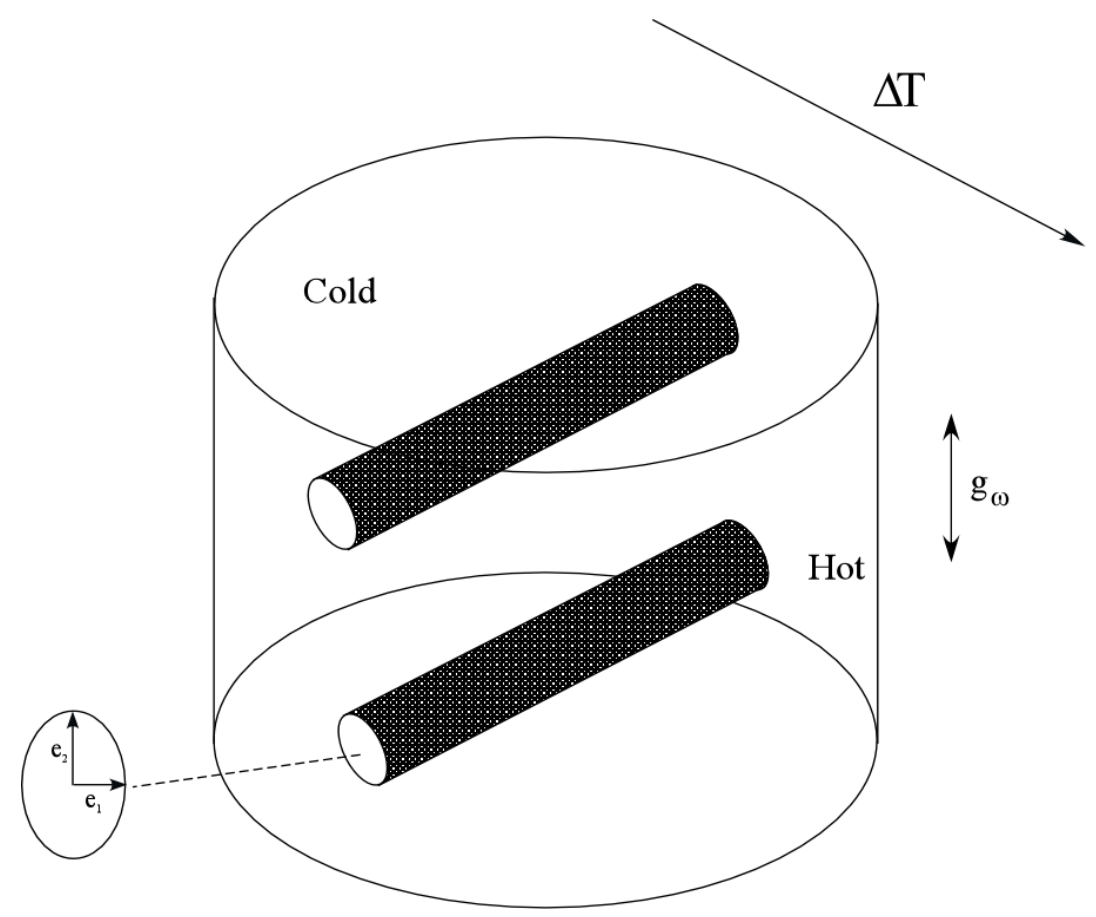

Figure 3: Sketch of cylindrical enclosure, related thermal boundary conditions, imposed vibrations and emerging particulate aggregates.

The outcomes of such a parametric investigation are summarized in Table II.

TABLE II: Grid refinement study: maximum of the velocity components of the $\underline{V}$ and $\langle\underline{V}\rangle$ and fields and size of the axes $\mathrm{e}_{1}$ and $\mathrm{e}_{2}$ shown in Fig. 3 as a function of mesh resolution (cylindrical enclosure with differentially heated sidewall and adiabatic ends, vibrations along the $\mathrm{z}$ axix, $\operatorname{Pr}=8$, $\left.\mathrm{A}=0.75, \varpi=1 \times 10^{3}, \mathrm{Ra}_{\omega}=1.58 \times 10^{4}, \mathrm{t}=0.56\right)$.

\begin{tabular}{llll}
\hline \hline $\mathrm{N}_{\mathrm{Z}} \mathrm{xN}_{\mathrm{r}} \mathrm{XN}_{\varphi}$ & $\mathrm{u}_{\max }$ & $\mathrm{v}_{\max }$ & $\mathrm{W}_{\max }$ \\
$20 \mathrm{x} 14 \mathrm{x} 20$ & 22.090459 & 23.988021 & 22.126823 \\
$27 \mathrm{x} 18 \mathrm{x} 27$ & 21.886765 & 23.898973 & 21.874763 \\
$41 \times 27 \times 41$ & 21.825312 & 23.857689 & 21.842886 \\
$49 \times 33 \times 49$ & 21.814679 & 23.835055 & 21.835362 \\
\hline \hline
\end{tabular}

\begin{tabular}{llllll}
\hline \hline Mesh & $\langle u\rangle_{\max }$ & $\langle v\rangle_{\max }$ & $\langle w\rangle_{\max }$ & $\mathrm{e}_{1} \times 2$ & $\mathrm{e}_{2} \times 2$ \\
20x14x20 & 0.3027287 & 0.3739038 & 0.2536782 & 0.082 & 0.234 \\
$27 \times 18 \times 27$ & 0.3023345 & 0.3685796 & 0.2423693 & 0.094 & 0.253 \\
$41 \times 27 \times 41$ & 0.3021813 & 0.3552482 & 0.2318292 & 0.118 & 0.270 \\
$49 \times 33 \times 49$ & 0.3020539 & 0.3531231 & 0.2296784 & 0.121 & 0.274 \\
\hline \hline
\end{tabular}

\begin{tabular}{llll}
\hline \hline $41 \times 27 \times 41$ & $\langle u\rangle_{\max }$ & $\langle v\rangle_{\max }$ & $\langle w\rangle_{\max }$ \\
Present code & 0.3021813 & 0.3552482 & 0.2318292 \\
Gershuni's & 0.2987363 & 0.3489834 & 0.2268736 \\
framework & & & \\
\hline \hline
\end{tabular}


Table IIa indicates that grid-independence of the flow of thermovibrational nature could be attained even with the coarsest mesh considered in the table (the values obtained with a grid $20 \times 14 \times 20$ match with a reasonable approximation $(<1 \%)$ the values obtained with finer grids). This result can be easily explained according to the nature of the emerging flow, which apart from being very slow, is regular and smooth in time and space with no thermal or kinematic boundary layers.

The velocities shown in Table II would correspond in dimensional form for a cylinder with height $2 \times 10^{-2} \mathrm{~m}$ to a maximum velocity $\cong 2 \times 10^{-4} \mathrm{~m} / \mathrm{s}$ and a maximum corresponding time-averaged velocity $\cong 2 \times 10^{-6} \mathrm{~m} / \mathrm{s}$ (among other things this also confirms our initial assumption about the timeaveraged convective effects of thermovibrational nature being very small with respect to the oscillatory component of fluid velocity, i.e. $\langle u\rangle_{\max } / u_{\max } \cong\langle v\rangle_{\max } / v_{\max } \cong\langle w\rangle_{\max } / w_{\max } \cong 10^{-2}$ ).

Nevertheless, Table IIb also clearly indicates that a finer mesh is required for attaining a reasonable level of convergence in the typical size of the resulting particle structures (a mesh with at least $\mathrm{N}_{\mathrm{Z}} \mathrm{XN}_{\mathrm{r}} \mathrm{xN} \mathrm{N}_{\varphi}=41 \times 27 \times 41$ points is required to make the percentage variation of $\mathrm{e}_{1}$ and $\mathrm{e}_{2}$ less than $3 \%$ with respect to the next row in the table). Additional validation for the present code was provided by the very good agreement between the time-averaged quantities obtained in the framework of the so-called Gershuni's approach discussed before (Table IIc). Figure 4, shows the time-averaged pattern in the yz plane, corresponding to the well-known "quadrupolar field" (Khallouf et al. [50], Mialdun et al. [51]) that emerges in closed cavities for vibrations perpendicular to the temperature gradient.
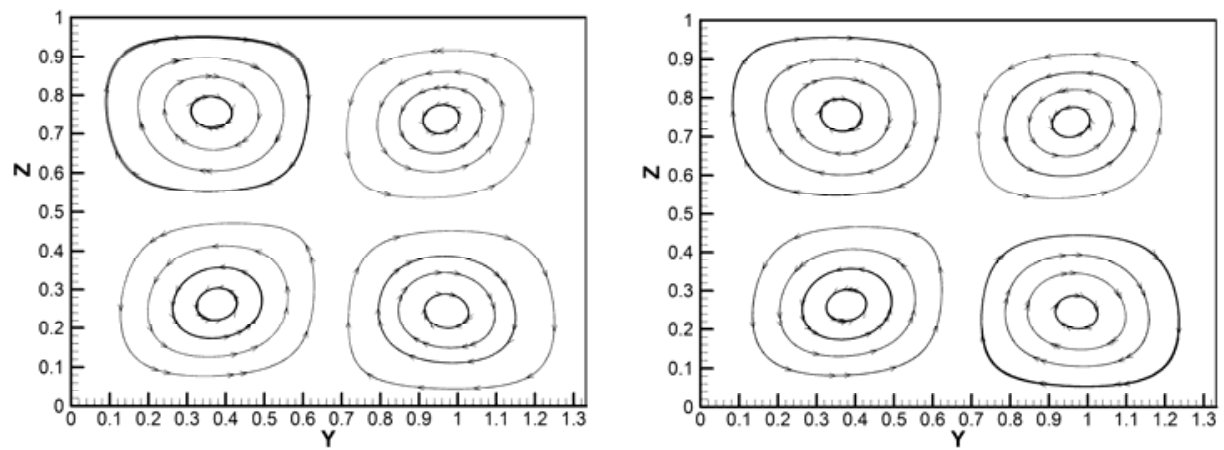

Figure 4: Classical "quadrupolar" flow field in the yz plane obtained by a) time-averaging of the oscillatory solution, b) integration of the Gershuni's equations (cylinder with insulated ends and nonisothermal sidewall with temperature varying as a sinusoidal function of the azimuthal angle, vibrations along the $\mathrm{z}$ axix, $\left.\mathrm{Pr}=8, \mathrm{~A}=0.75, \varpi=1 \times 10^{3}, \mathrm{Ra}_{\omega}=1.58 \times 10^{4}\right)$.

\section{Results}

The author's earlier analysis [12] was entirely concentrated on a geometrical configuration with straight walls (a cubical cavity) and a fixed direction of the temperature gradient, allowing most of the involved parameters (the amplitude and angular frequency of the acceleration disturbance, the characteristic nondimensional number $\mathrm{Ra}_{\omega}$ and the particle Stokes number to span relatively wide 
ranges). As the focus here is on the influence of curved walls in producing new specific (previously unseen) behaviours, we keep constant most of those parameters $\left(\operatorname{Pr}=8, A=0.75, \varpi=1 \times 10^{3}\right.$, $\operatorname{Ra}_{\omega}=1.58 \times 10^{4}, \gamma=1.5 \times 10^{6}$, and $\eta=1 \times 10^{-4}$ ) while allowing the specific forcing considered (direction of the imposed temperature gradient and associated perpendicular vibrations) to have different orientations in space (as shown in Fig. 1). To track the system evolution, solid particles $\left(\cong 4 \times 10^{4}\right.$ ) are initially seeded uniformly into the computational domain assuming their velocity equal to zero (to reflect the quiescent conditions of the surrounding liquid).

\section{A. Differentially heated ends and adiabatic sidewall}

\section{The Thermofluid-dynamic Field}

Figures 5 and 6 show the spatio-temporal evolution of the velocity and temperature field during half of a cycle of forcing in the case (a) of a temperature gradient parallel to the system symmetry axis, insulated sidewall and vibrations aligned with the $\mathrm{x}$ axis. It is worth starting the related discussion from the relatively simple observation that while, as expected (due to the relatively small value of the Gershuni number), the departure of the temperature field from purely diffusive conditions (a purely linear distribution of temperature along the $\mathrm{z}$ axis) is relatively weak (see Figure 5), welldefined convective structures are visible at any instant in any cross section $z=$ const (e.g., $z=0$, see Figure 6).
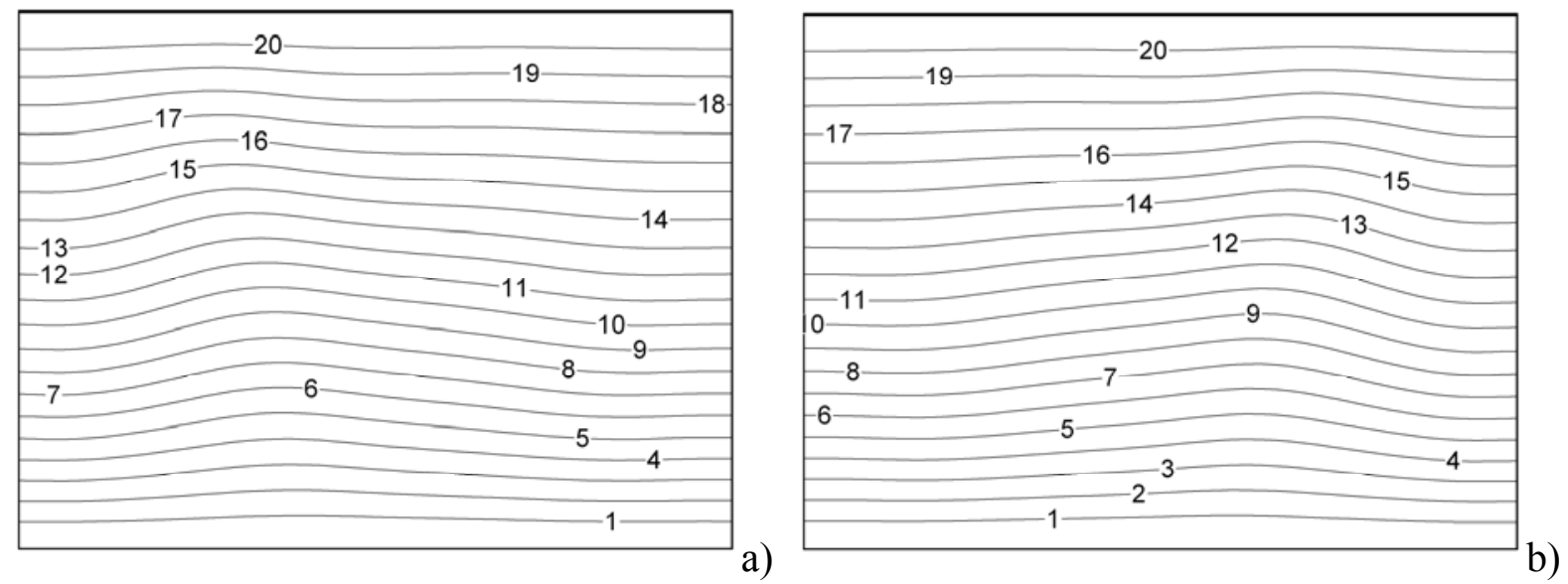

Figure 5: Snapshots of temperature distribution in the meridian plane $\mathrm{xz}(\varphi=0)$ at two instants during the period $\tau$ of oscillation (case A, contour legend: level $1 \rightarrow \mathrm{T}=-0.45$, level $20 \rightarrow \mathrm{T}=0.45$, $\left.\Delta_{\text {level }} \rightarrow 0.05\right)$ : a) $\left.\mathrm{t}=\mathrm{t}_{0}, \mathrm{~b}\right) \mathrm{t}=\mathrm{t}_{0}+\tau / 2(\tau=2 \pi / \varpi)$. 

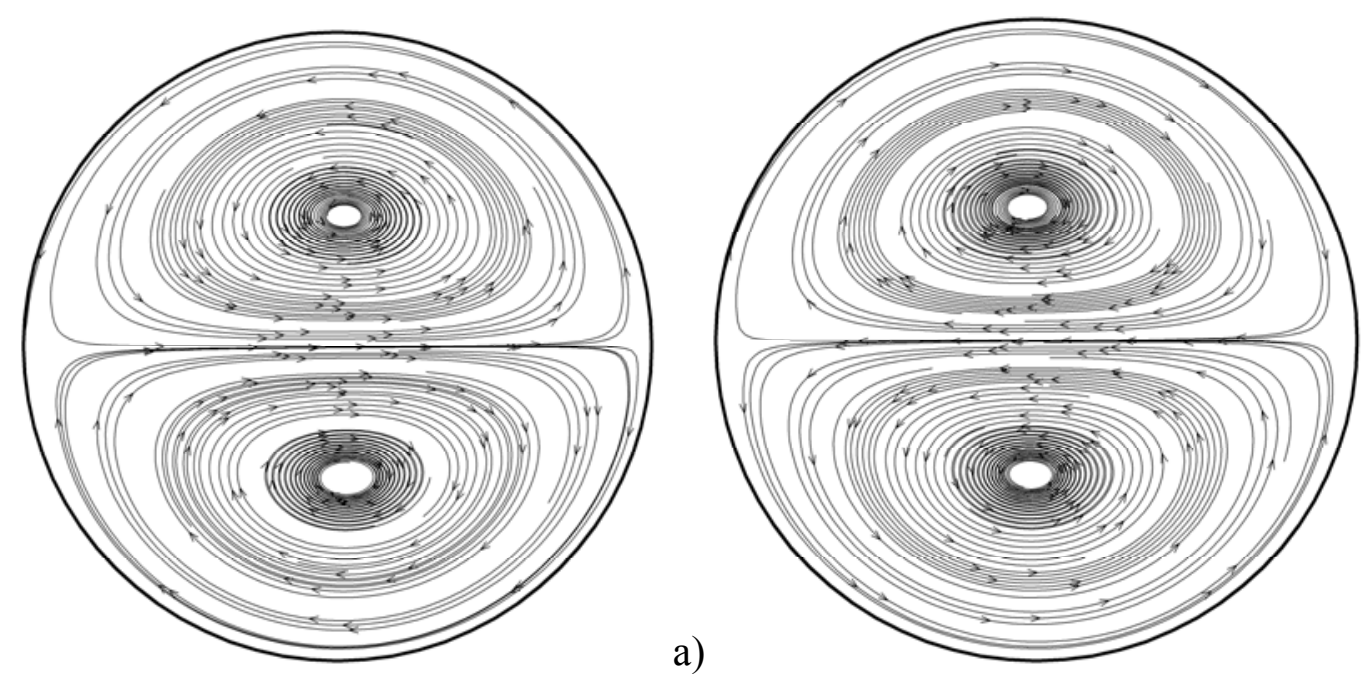

b)

Figure 6: Snapshots of velocity field in the cross section $z=0$ at two instants during the period $\tau$ of oscillation (case A): a) $\left.\mathrm{t}=\mathrm{t}_{0}, \mathrm{~b}\right) \mathrm{t}=\mathrm{t}_{0}+\tau / 2(\tau=2 \pi / \varpi)$.

In practice, regardless of the considered time, convective flow consists essentially of two main rolls with axis extended along the $\mathrm{z}$ direction, one being the mirror image of the other and sense of circulation depending on the specific instant considered within the cycle of forcing.

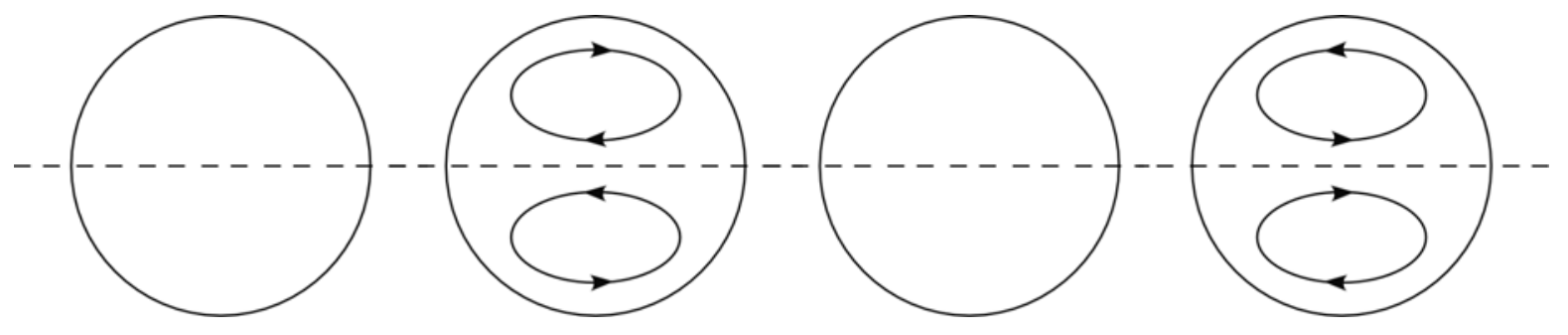

Figure 7: Sequence (sketch) showing the evolution of vibrations-induced convective cells in the generic cross section $\mathrm{z}=$ const during one period of oscillation (case A).

The intersection of such axial rolls with cross-sections $\mathrm{z}=$ const produces two apparent cells, one clockwise and the other anticlockwise oriented. These convective cells are not stationary and change their sense of rotation periodically (their intensity is not constant in time; if the considered convective cell is clockwise oriented during the first half-period of oscillation, then during the second half it vanishes and finally reappears in the same position anticlockwise oriented, see Fig. 7). 

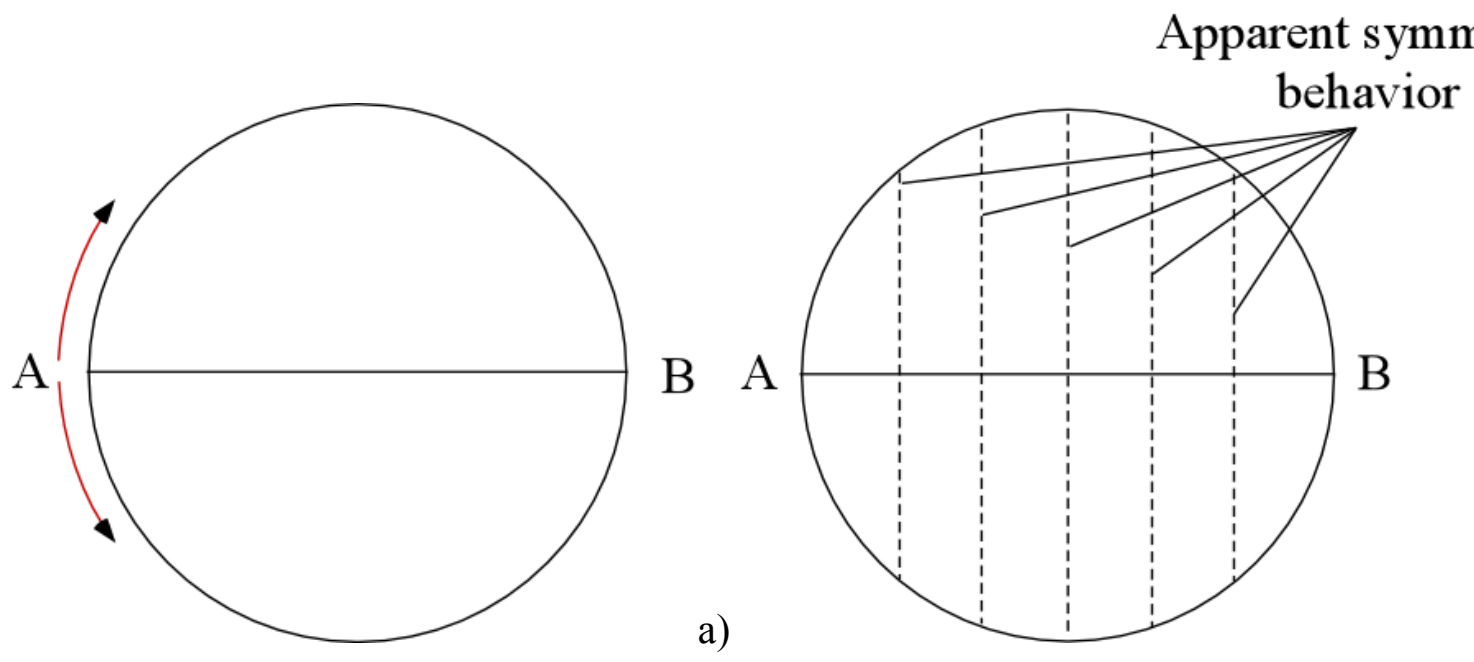

b)

Figure 8: Mental divisibility (topology) of the flow configuration (sketch)

Therefore, a clear distinguishing mark of this convective mode is the mental divisibility (topology) of the flow configuration in two semicircular regions (such a divisibility can be sketched, as shown in Figure 8, by introducing an ideal plane of symmetry $\mathrm{AB}$, hereafter referred to as PS, that separates the flow configuration into two sectors).

At one end of PS two opposite azimuthal currents originate, and at the other end of PS they vanish. Assuming that at a given instant $t=0$ the starting point of such counterpropagating flows is the point A on the left side, two initially very close parcels of fluid located there at $\varphi=\pi^{+}$and $\varphi=\pi^{-}$, respectively, (see Fig. 8a) will move towards the right side (point B) as $t$ increases, one in a clockwise direction (top semi-circumference in Fig. 8a) and the other anticlockwise (bottom semicircumference in Fig. 8a). In particular, both parcels will move with identical and constant velocity and after a duration of one half of the vibration frequency $[t=1 /(2 f)]$ they reunite at the opposite side of point A (point B).

At time $\mathrm{t}=1 / f$, however, as the time-varying acceleration induced by vibrations changes sign, the same process will be repeated with two parcels initially located at $\varphi=0^{-}$and $\varphi=\pi^{+}$, respectively, moving towards the left side in a clockwise direction along bottom semi-circumference and in the anticlockwise direction along the top semi-circumference. 

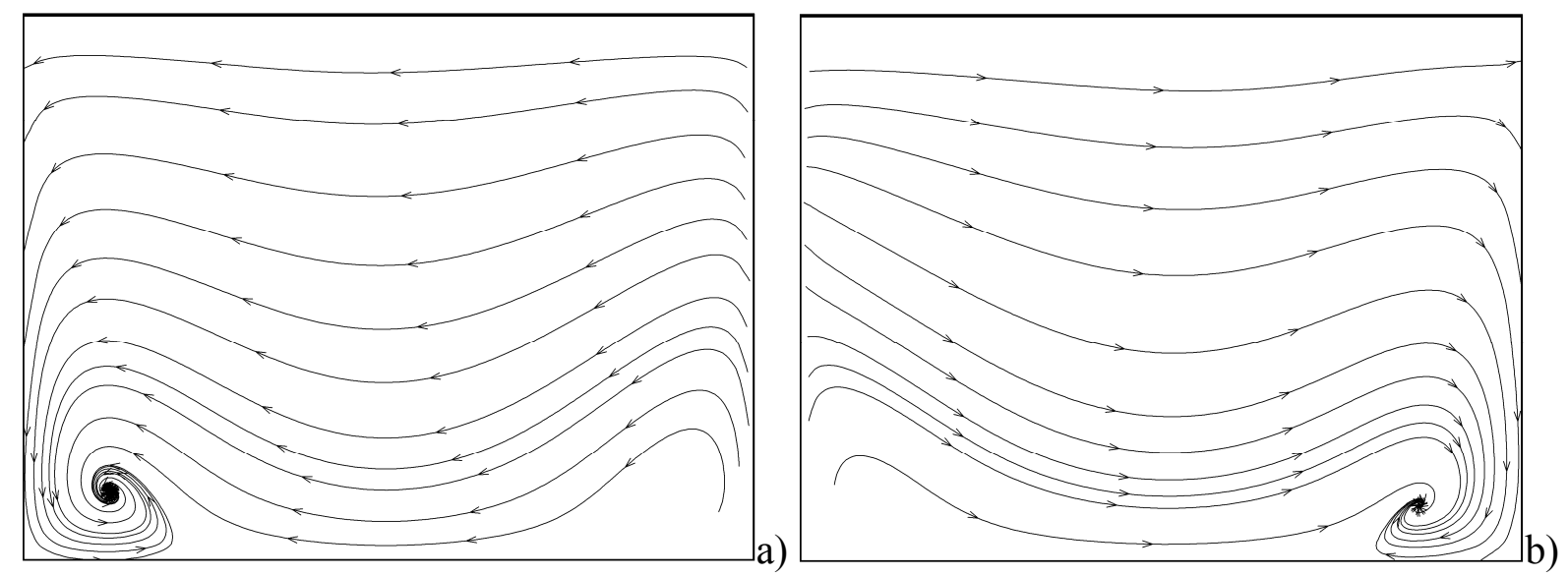

Figure 9: Snapshots of velocity field in the meridian plane $\mathrm{xz}(\varphi=0)$ at two instants during the period $\tau$ of oscillation (case A): a) $\left.\mathrm{t}=\mathrm{t}_{0}, \mathrm{~b}\right) \mathrm{t}=\mathrm{t}_{0}+\tau / 2(\tau=2 \pi / \varpi)$.

As a natural consequence of such a mechanism, the instantaneous return flow will tend to be aligned with the $\mathrm{AB}$ direction (i.e. with the direction of imposed vibrations) and the overall flow will display a mirror symmetry with respect to this direction in any section perpendicular to PS (whereas it will appear strongly asymmetric in any section parallel to the x axis, Fig. 9).

\section{Particle Structures}

As shown in Fig. 10, after a relatively long transient time, well-defined particle structures are formed in the cylinder. Particles are no longer uniformly spaced in the liquid, rather they tend to accumulate on spatially extended surfaces located in proximity to the lateral sidewall.

Most surprisingly, although such structures tend to retain the mirror symmetry with respect to the plane $\mathrm{AB}$ (the $\mathrm{x}$ axis, i.e. the direction of applied vibrations), and to reflect the typical topology of flow induced by vibrations (with fluid moving alternatively back and forth along the sidewall between points A and B), some regions completely depleted of particles appear periodically in the cylinder. Such zones are separated from regions in which solid particles are more or less uniformly dispersed by the aforementioned spatially extended surfaces along which particles undergo preferential accumulation.

The subsequent evolution consists essentially of a rhythmic left-right displacement of the overall pattern and related characteristic independent loops visible in cross sections, which means that the system response to the imposed vibration is synchronous (or harmonic) (particles and velocity fields oscillating at the same frequency of the imposed sinusoidal forcing). 

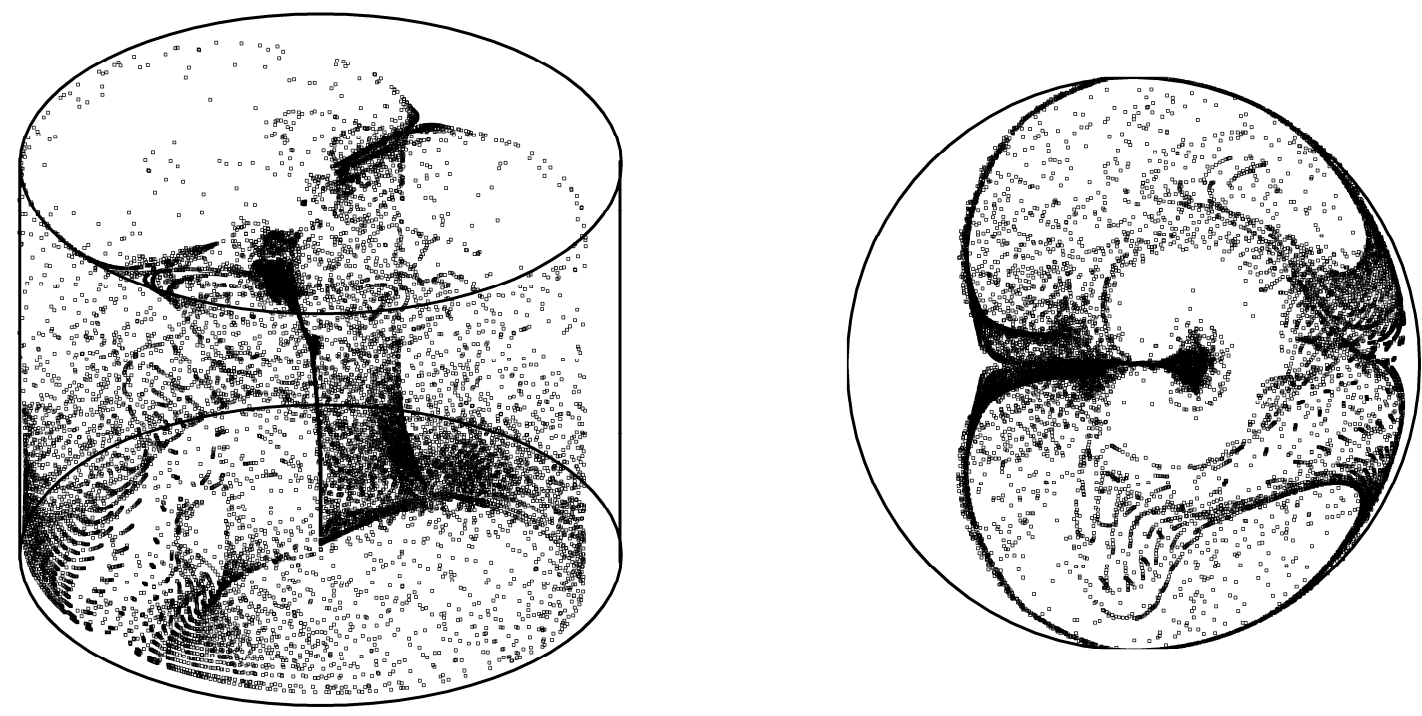

a)
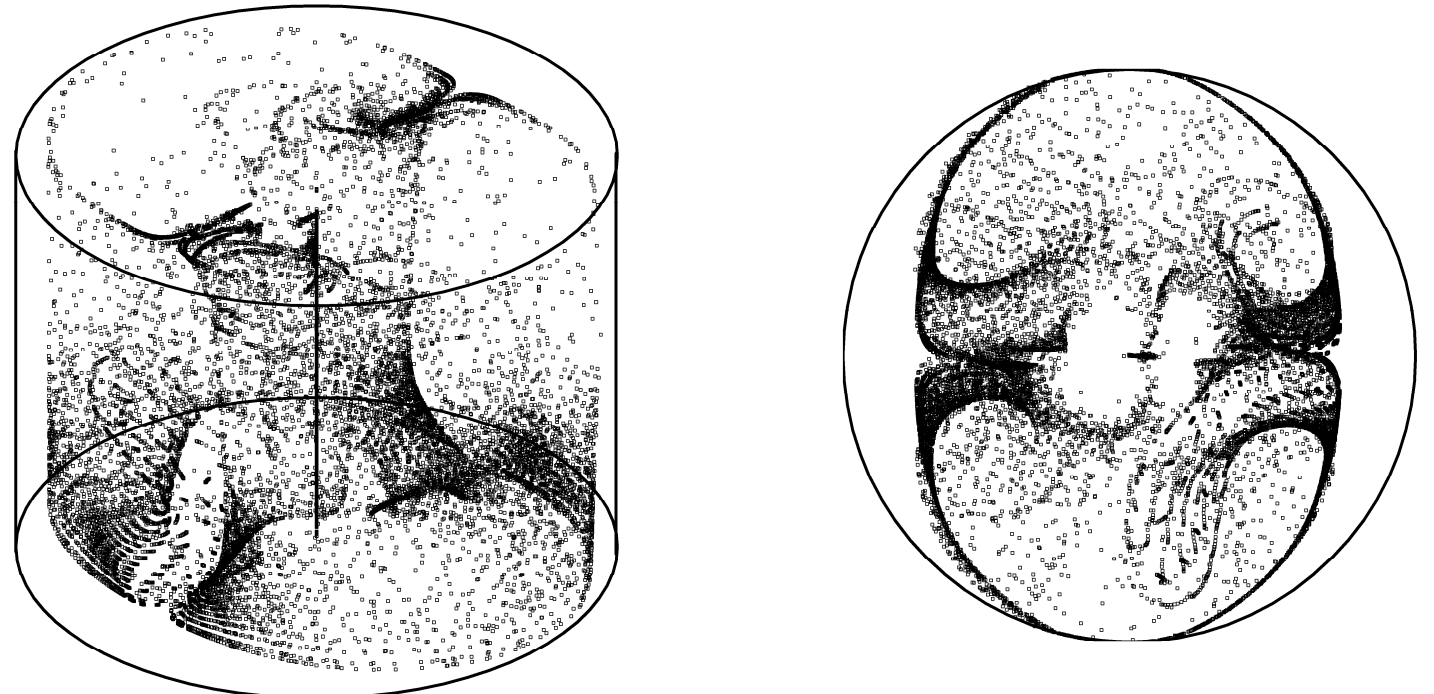

b)
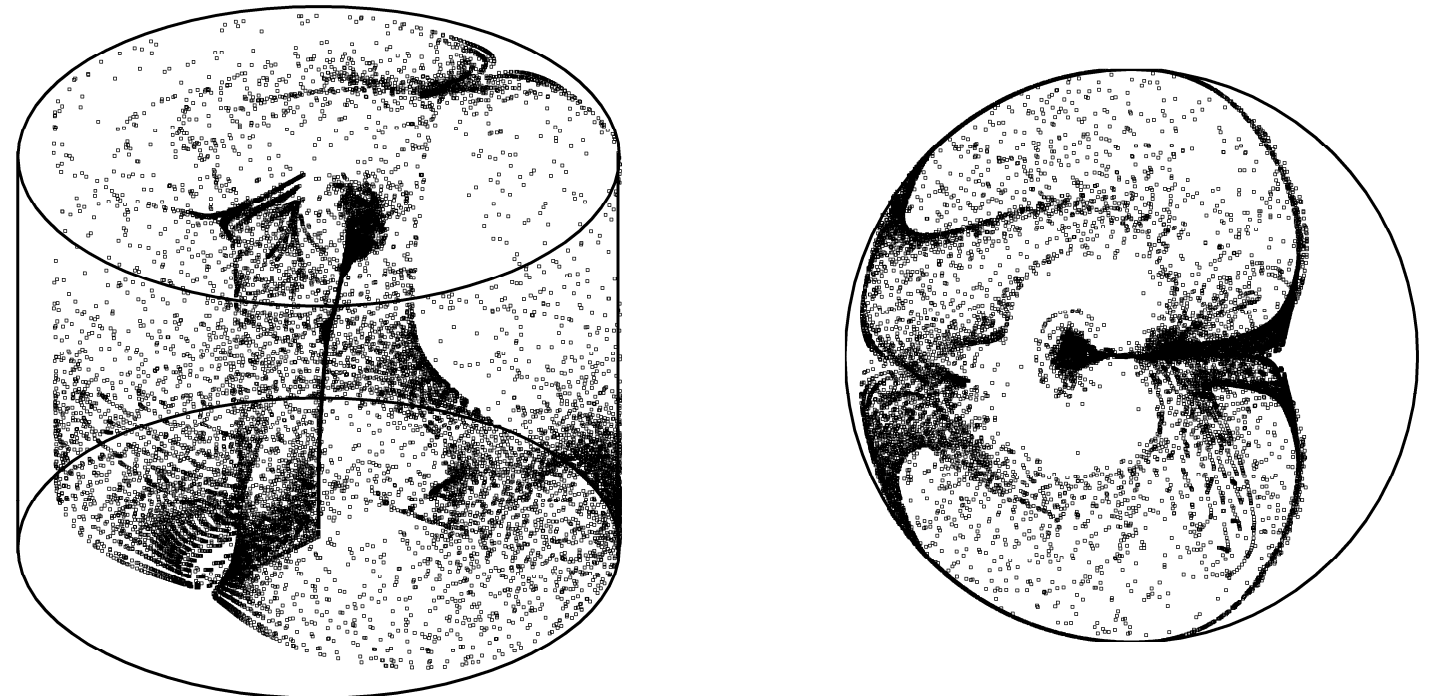

c)

Figure 10: $3 D$ snapshots of particle aggregates (case A): a) $\left.\left.t=t_{0}, b\right) t=t_{0}+\tau / 4, c\right) t=t_{0}+\tau / 2\left(t_{0}=1.4 \times 10^{-}\right.$ $1, \tau=2 \pi / \varpi)$. 
In other words, once the particle-dense surfaces are formed, a further increase of time produces no variation in the morphological and topological properties of the pattern. Under the effect of vibrations the pattern oscillates back and forth along the direction of vibrations essentially as a whole (compare, e.g., Figs 10a and 10c, although the overall pattern is shifted to the right in Fig. 10a and to the left in Fig. 10c, the characteristic size of the two circuits and their distance remain constant) at the vibration frequency as experienced by an observer in the laboratory (fixed) reference frame.

An interesting behavior, deserving some additional discussion is evident along the line $\mathrm{AB}$ (see Figure 10, right part). A dark region (particle-dense spot) where particles accumulate is visible near the symmetry axis in the right part of Fig. 10a and 10c (no dark region is visible in Fig. 10b).

This process can be explained by resorting to an analogy with similar pluming phenomena in thermal convection. Just as in classical thermal-plume formation mechanisms, the heated fluid rises due to buoyancy along a vertical line, producing a cap on top as a result of the drag exerted on it by the overlying fluid (the result in the temperature map is something that looks like a "mushroom"), here the strong alternating flow along the line AB, produces periodically a "plume of particles" moving from left to right or in the opposite direction. The concentration of particles in the "plume cap" is due essentially to the inertia that tends to slow down their motion with respect to the carrier liquid. In line with such an analogy, this "plume" effect disappears when the fluid velocity along $\mathrm{AB}$ is weakened (Fig. 10b).

\section{B. Non isothermal sidewall and adiabatic bases}

\section{The Thermofluid-dynamic Field}

Figure 11 shows that the flow structure changes completely when the adiabatic lateral boundary is replaced by a non-isothermal sidewall (with a resulting temperature gradient along the y direction and perpendicular accelerations, i.e. vibrations aligned with the $\mathrm{z}$ axis).

Given the relatively small value of the Gershuni number, the temperature distribution is still approximately linear inside the cylindrical enclosure (Fig. 11, left). The convective rolls with axis parallel to $z$, alternating their sense of rotation as time passes that we have seen in Sect. IIIA, however, are no longer a feature of the flow. Rather (Fig. 11, right, to be compared with Fig. 9), it displays a single main roll with axis parallel to $\mathrm{x}$, changing cyclically its sense of rotation (from the clockwise sense to the anticlockwise one for increasing values of $t$ ). Such a main roll is produced essentially by the interplay between the temperature distribution and the buoyancy effect originating from the vibration-induced acceleration. More precisely, vibration-induced buoyancy and the imposed temperature gradient alternatively cooperate to produce a clockwise or anticlockwise convective cell over a cycle of the modulation. In that part of the cycle where the acceleration is directed along the positive $\mathrm{x}$ axis direction, the resulting convective cell will be anticlockwise oriented, vice versa (clockwise oriented roll when $g_{\omega}(t)$ changes its sign). The concept of mental 
divisibility of the flow configuration in two mirror regions is still applicable, but, obviously, in this case the ideal plane of symmetry coincides with the xz plane.

\section{Particle Structures}

As shown in Fig. 12, after a relatively long transient time, well-defined particle patterns are formed in the cylinder. Particles are no longer uniformly spaced in the liquid, and as in the case treated in Sect. IIIA, they tend to accumulate on spatially extended surfaces.

Most surprisingly, however, the volume percentage occupied by such surfaces is relatively small in comparison to the companion case of Sect. IIIA. Two distinct tubular structures can be recognized in Fig. 12 very similar to those that would be obtained in the case of a cubical cavity [12]. Their overall radial extension is approximately equal to the diameter of the cylindrical enclosure and their orientation is parallel to the direction of imposed vibrations (the $\mathrm{x}$ axis). The two surfaces, however are well separated (their axial distance is approximately $40 \%$ of the cylinder height). Both tubular structures have an elliptic-shaped section (obtained by intersection with the yz plane). The characteristic sizes of their elliptic section $e_{1}$ and $e_{2}$ are only $12 \%$ and $26 \%$ of the cylinder axial extension, which may be regarded as a clear distinguishing mark with respect to the situation considered in Sect. IIIA (obviously, another clear difference is given by the shape of the section per se, as the section shape of the tubular structures shown in Fig. 10, was more similar to an orange slice than to an ellipse).
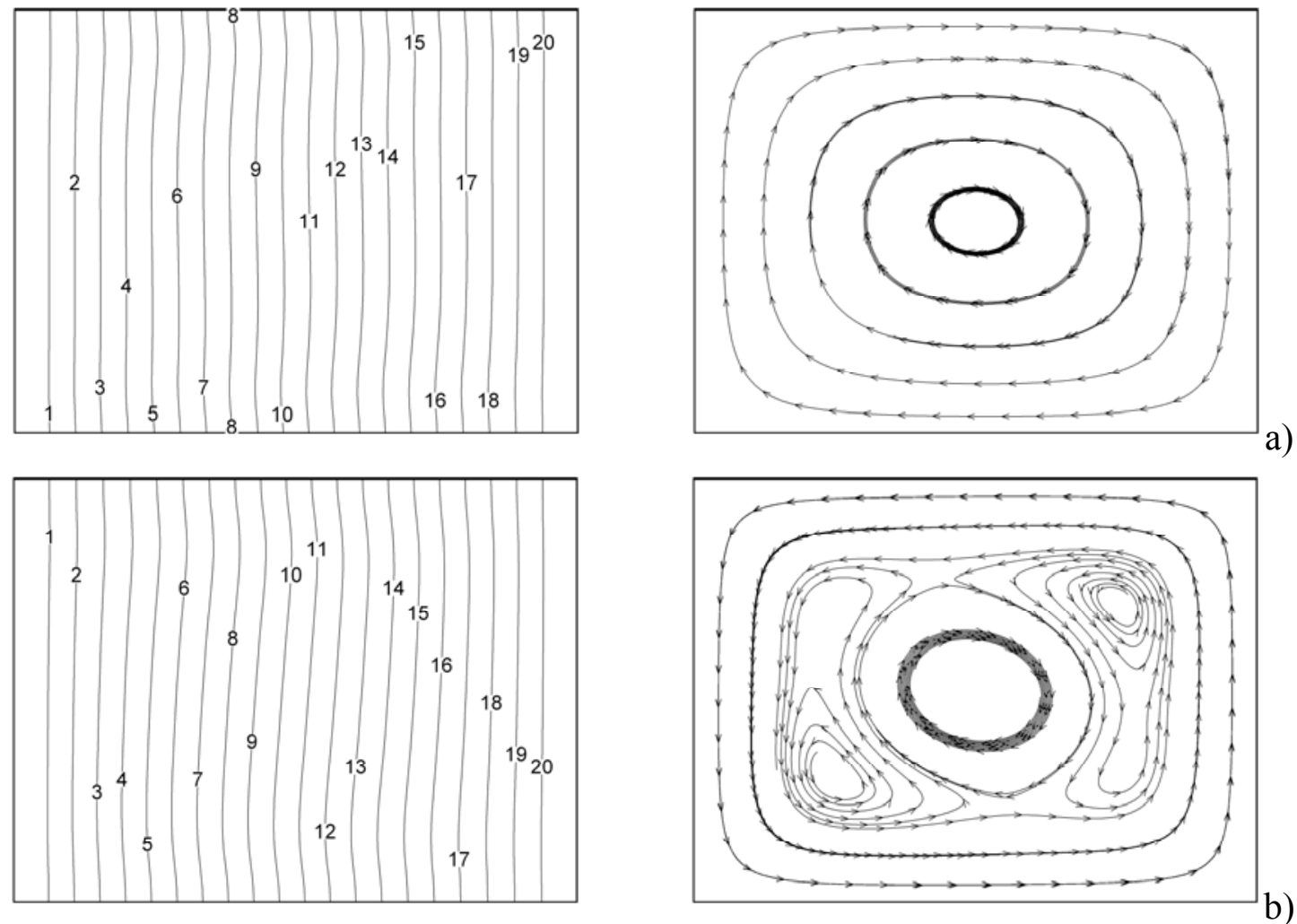

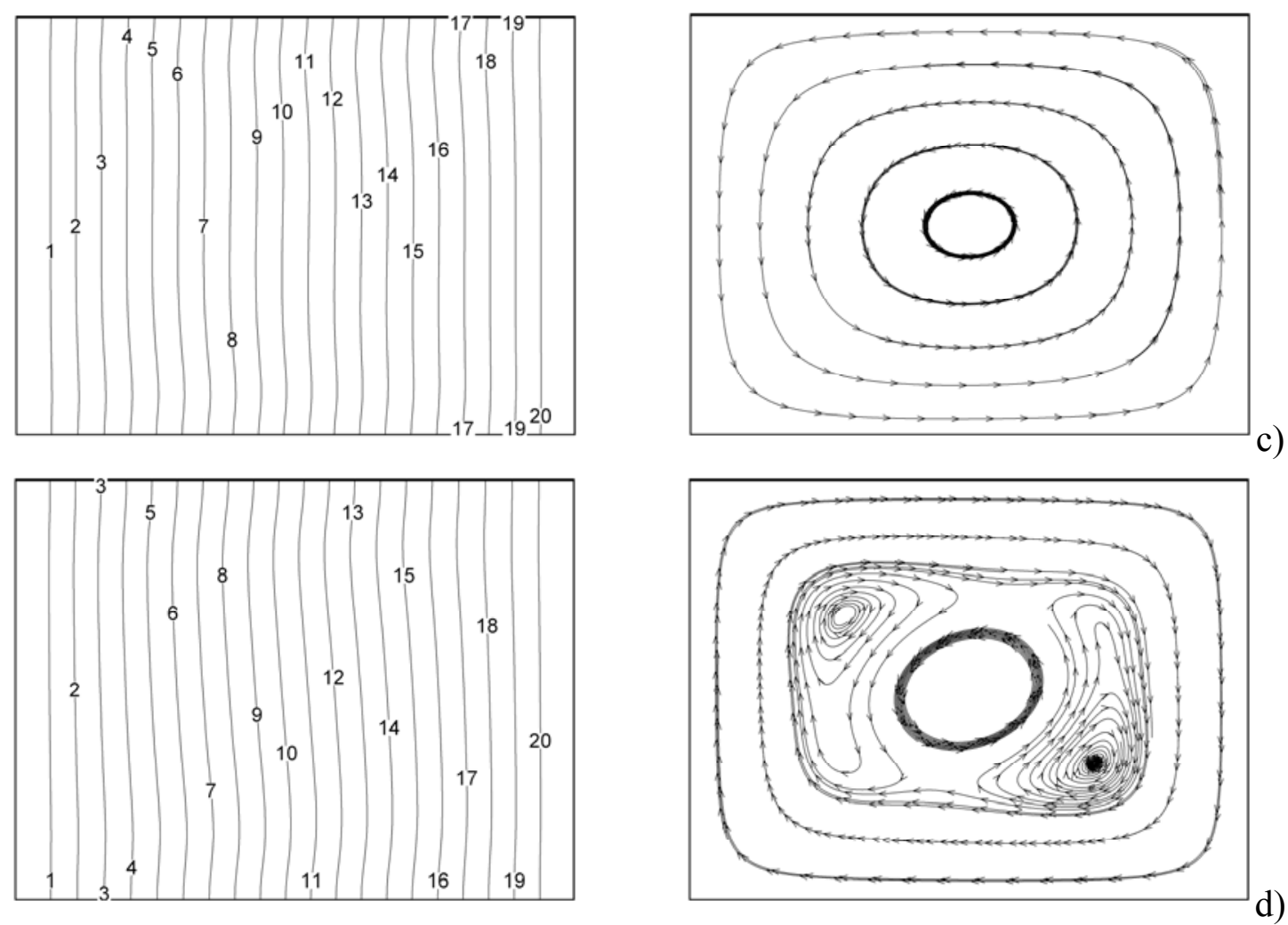

Figure 11: Snapshots of temperature distribution (left) and velocity field (right) in the meridian plane yz $(\varphi=\pi / 2)$ at four instants evenly space in time during the period $\tau$ of oscillation (case $B$, contour legend: level $1 \rightarrow \mathrm{T}=-0.45$, level $\left.20 \rightarrow \mathrm{T}=0.45, \Delta_{\text {level }} \rightarrow 0.05\right):$ a) $\left.\mathrm{t}=\mathrm{t}_{0}, \mathrm{~b}\right) \mathrm{t}=\mathrm{t}_{0}+\tau / 4$, c) $\mathrm{t}=\mathrm{t}_{0}+\tau$ $/ 4, d) \mathrm{t}=\mathrm{t}_{0}+3 \tau / 4 \quad(\tau=2 \pi / \varpi)$.

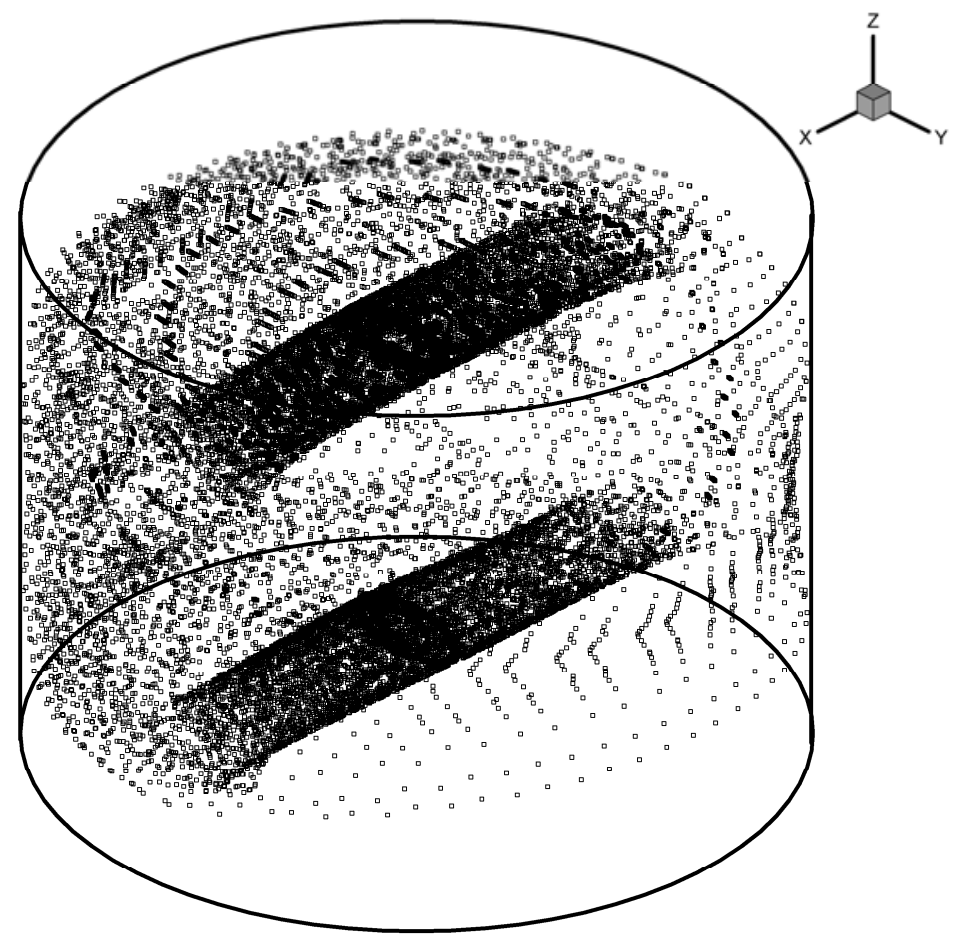

Figure 12: $3 \mathrm{D}$ snapshots of particle aggregates (case $\mathrm{B}, \mathrm{t}=5.6 \times 10^{-1}$ ). 



Figure 13: Lateral view (from the $\mathrm{x}$ axis perspective) of particle aggregates as a function of time (case B): a) $\mathrm{t}=2.7 \times 10^{-2}$, b) $\mathrm{t}=9.5 \times 10^{-2}$, c) $\mathrm{t}=1.6 \times 10^{-1}$, d) $\mathrm{t}=1.9 \times 10^{-1}$, e) $\left.\mathrm{t}=2.24 \times 10^{-1}, \mathrm{f}\right) \mathrm{t}=2.56 \times 10^{-1}$.

To fully understand the mechanism at work in this case (and appreciate the related theoretical implications), however, we have to examine in detail the spatio-temporal interplay established between buoyancy-induced flow and particle aggregates. 
It is indeed the mechanism produced by the instantaneous (oscillating in time) flow (Fig. 11, right) in combination with vibration-induced particle time-displacements, whose nonlinear effects on particles accumulate over time, which leads to the observed particulate pattern. This is clearly illustrated in Fig. 13, which shows the stages of evolution of the considered system from the initial condition with particles uniformly distributed in the fluid up to the emergence of the final pattern with tubular structures aligned with the $\mathrm{x}$ axis.

At the beginning (Fig. 13a) particle close to the walls tend to be accumulated owing to edge effects into two separate surfaces extended along the direction perpendicular to the imposed vibrations. In practice, this is due to the fact that when a group of particles moving under the effect of the vibration-induced periodic force (along $\mathrm{z}$ ) comes in proximity to one of the two cylinder bases, particles tend all to be accumulated along the boundary direction owing to the well-known impenetrability property of solid walls. This is reflected in the formation of an apparently marked (particle-dense) line in the lateral view shown in Fig. 13.

When the periodic force changes its sign and particles are moved in the opposite direction, the above process results in the appearance of a particle-dense line separating the region containing particles from the pure liquid (particle-depleted region).

One must keep in mind, however, that, as illustrated in Sect. IIIB1, the influence of vibrations is not limited to the periodic force exerted on particles along the $\mathrm{z}$ direction. They also produce flow of thermovibrational nature (Fig. 11) that can transport particles. This is essentially the reason why the abovementioned particle-dense surfaces produced by the interaction with solid ends tend to be stretched and folded as time passes.

The mechanism of pattern formation consists basically of a progressive translation away from the walls of such two apparent marked lines and ensuing folding of their part located in the cold region of the plane towards the interior (Fig. 13b). Such a folding is due essentially to the instantaneous motion of fluid shown in Fig. 11, which is alternatively accelerated from the hot to the cold side (due to buoyancy) along the top or bottom wall (depending on the instantaneous direction of acceleration) and then released in the cold region of the cylindrical enclosure. This process is responsible for the formation in the initial stages of evolution of a horizontal amphora-like shape as seen in the yz plane (the distribution of particles results in a shape that bulges out above $y=0$ and necks in below it).

As time increases, the progressive folding of the lines delimiting the region with particles produces two rounded patches "protruding" from the main particle region towards the cold side.

Figure $13 \mathrm{c}$ and $13 \mathrm{~d}$ show that, as time increases, the extension of the two small areas hanging out from the main particle region becomes increasingly elongated (along the y direction) and contracts until a neck is formed for each of them (Fig. 13e). As time is further increased, the size of such a neck shrinks spontaneously and finally the protuberant regions detach from the (mother) particle region (Fig. 13f) forming two independent loops (tubular structures).

Therefore, the following main stages of evolution can be identified in the process leading to the formation of the pattern: Stage I - the reason for the formation of a region where particles tend to 
concentrate is due to the interaction of particles with boundaries and the well-known impenetrability property of solid walls; Stage II - the changes in the shape of the boundary separating the region with particles from the particle-depleted space are due to the convective particle transport produced by the instantaneous flow of thermovibrational nature; such changes lead to the formation of two elongated particle regions protruding in the particle-depleted space; Stage III - this begins when, owing to convective effects, the axial extension of the protuberant regions becomes very small at a certain location along $y$; for a further increase in time, the size of the neck decreases rapidly to zero forming two well-defined tubular surfaces visible in Fig. 12 (whose intersection with the yz plane has approximately an elliptic shape).

The recognizable shapes formed by particles then tend to be stable in time. Indeed, the subsequent evolution consists essentially of a further depletion of the mother particle region with ensuing accumulation of particles in the two closed loops and a rhythmic (synchronous) top-bottom displacement of the overall pattern along the $\mathrm{z}$ direction. As in the companion case considered in Sect. IIIA, once the two particle circuits are formed, a further increase of time, produces no variation in the morphological and topological properties of the pattern, which undergoes rhythmic displacement along the $\mathrm{z}$ direction as an apparently solid unit (although, at the risk of repetition, we should recall again that the property of all particle pertaining to the pattern of moving as they would pertain to solid lattice is just an illusion).

\section{Inclined temperature gradient}

For the sake of completeness, in this section we finally discuss the case with inclined temperature gradient, which may be regarded as a hybrid configuration bridging the gap between the two limit conditions presented in the earlier sections.

\section{The Thermofluid-dynamic Field}

Given the relatively small value of the Gershuni number, the temperature distribution is still approximately linear inside the cylindrical enclosure (it is characterized by isotherms inclined with respect to the axial direction and uniformly distributed in space, Fig. 14, left). The velocity field, however, displays a structure much more complex than those obtained for the other considered heating conditions. This time, clearly distinguishable convective cells can be observed in both the $\mathrm{xz}$ plane and in sections perpendicular to the cylinder axis. In the latter case, in particular, four distinct counter-rotating cells can be identified (Figs. 15 and 16). Like the case considered in Sect. IIIA, such cells are not steady in time and change sense of rotation periodically. 

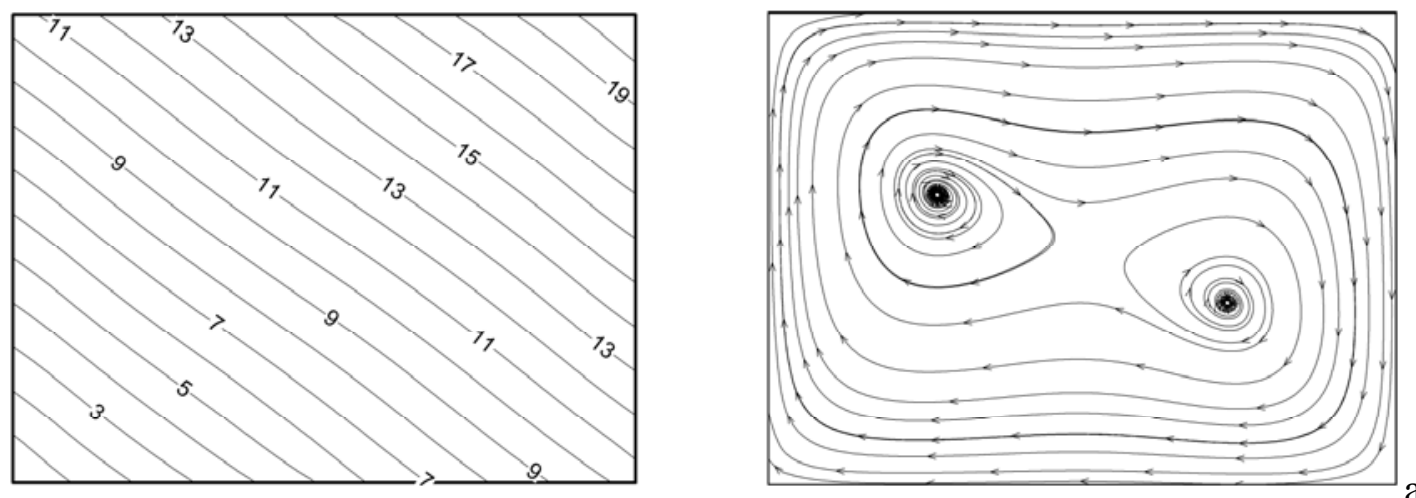

a)
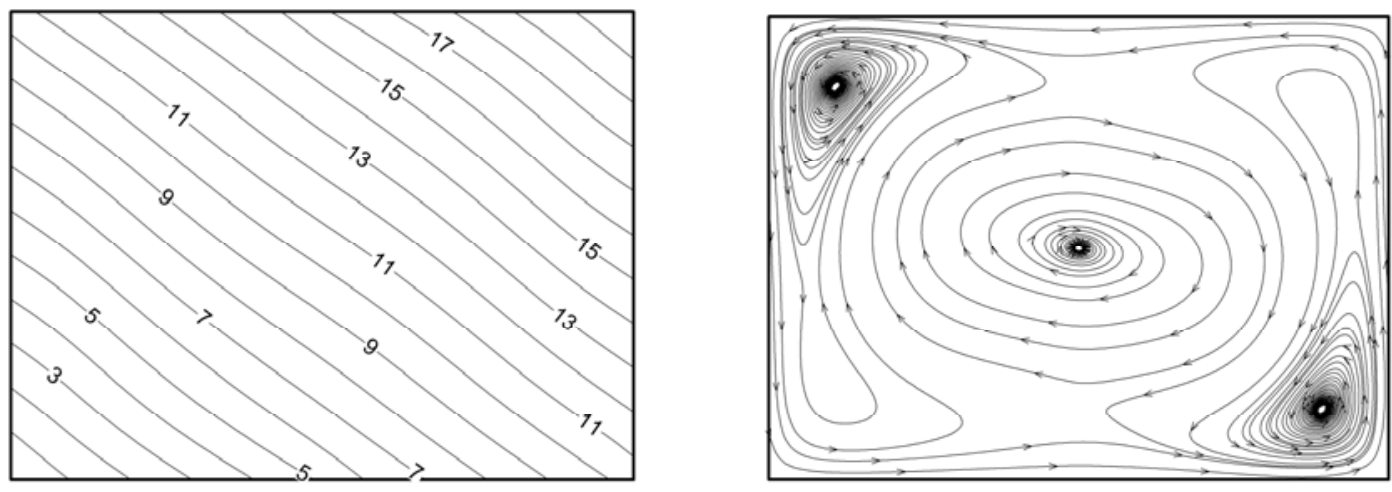

b)
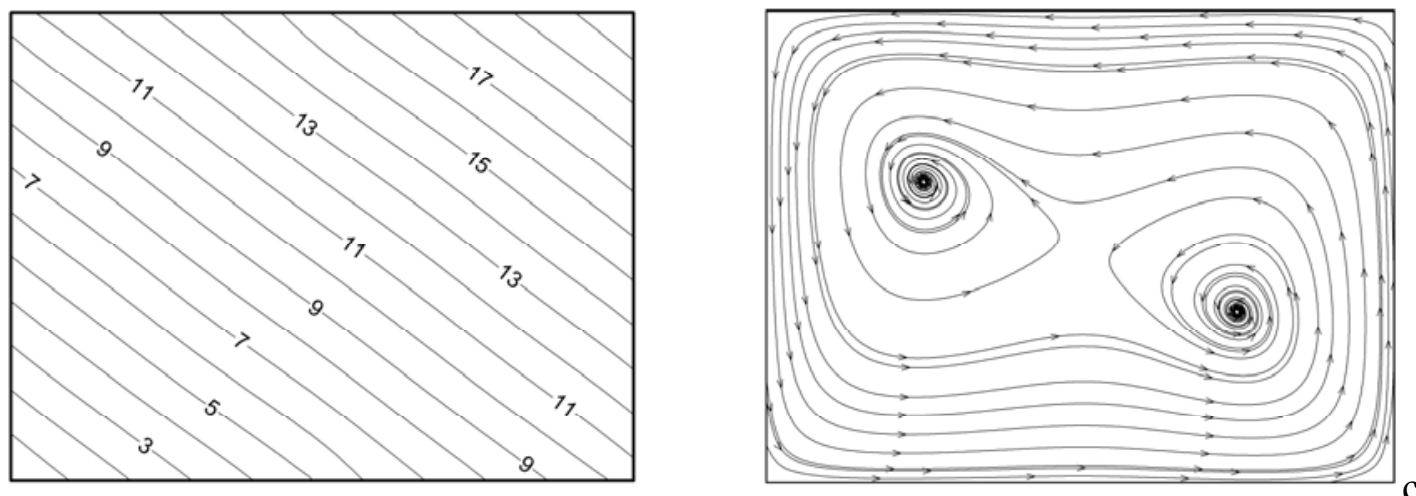

c)
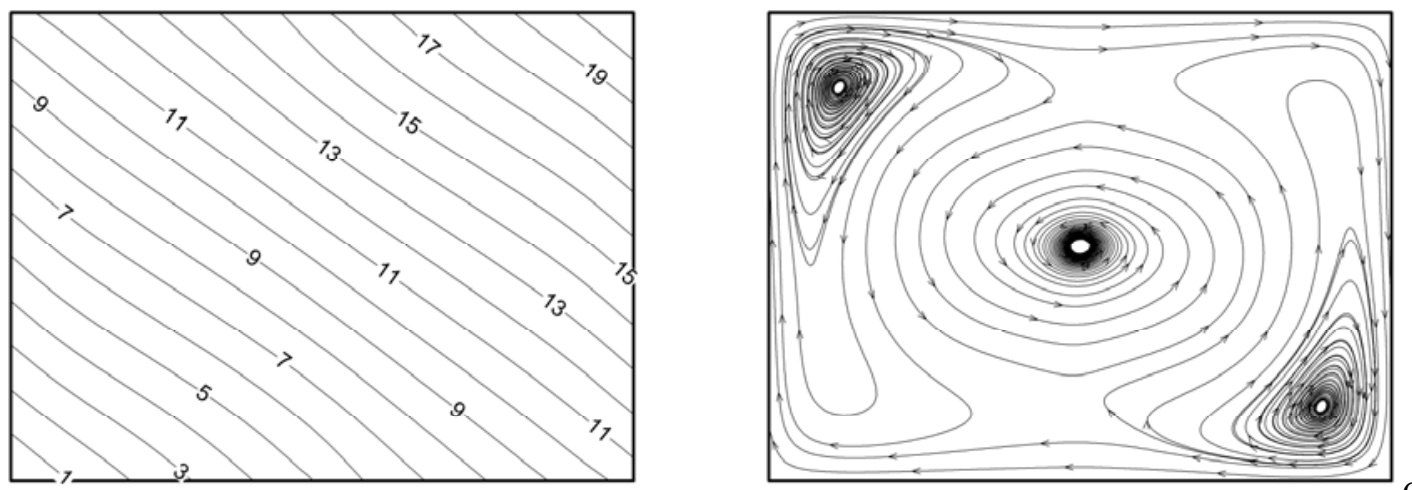

d)

Figure 14: Snapshots of temperature distribution (left) and velocity field (right) in the meridian plane $\mathrm{xz}(\varphi=0)$ at four instants evenly space in time during the period $\tau$ of oscillation (case $\mathrm{C}$, contour legend: level $1 \rightarrow \mathrm{T}=-0.45$, level $\left.20 \rightarrow \mathrm{T}=0.45, \Delta_{\text {level }} \rightarrow 0.05\right):$ a) $\left.\mathrm{t}=\mathrm{t}_{0}, \mathrm{~b}\right) \mathrm{t}=\mathrm{t}_{0}+\tau / 4$, c) $\mathrm{t}=\mathrm{t}_{0}+\tau$ $/ 4, \mathrm{~d}) \mathrm{t}=\mathrm{t}_{0}+3 \tau / 4 \quad(\tau=2 \pi / \varpi)$. 

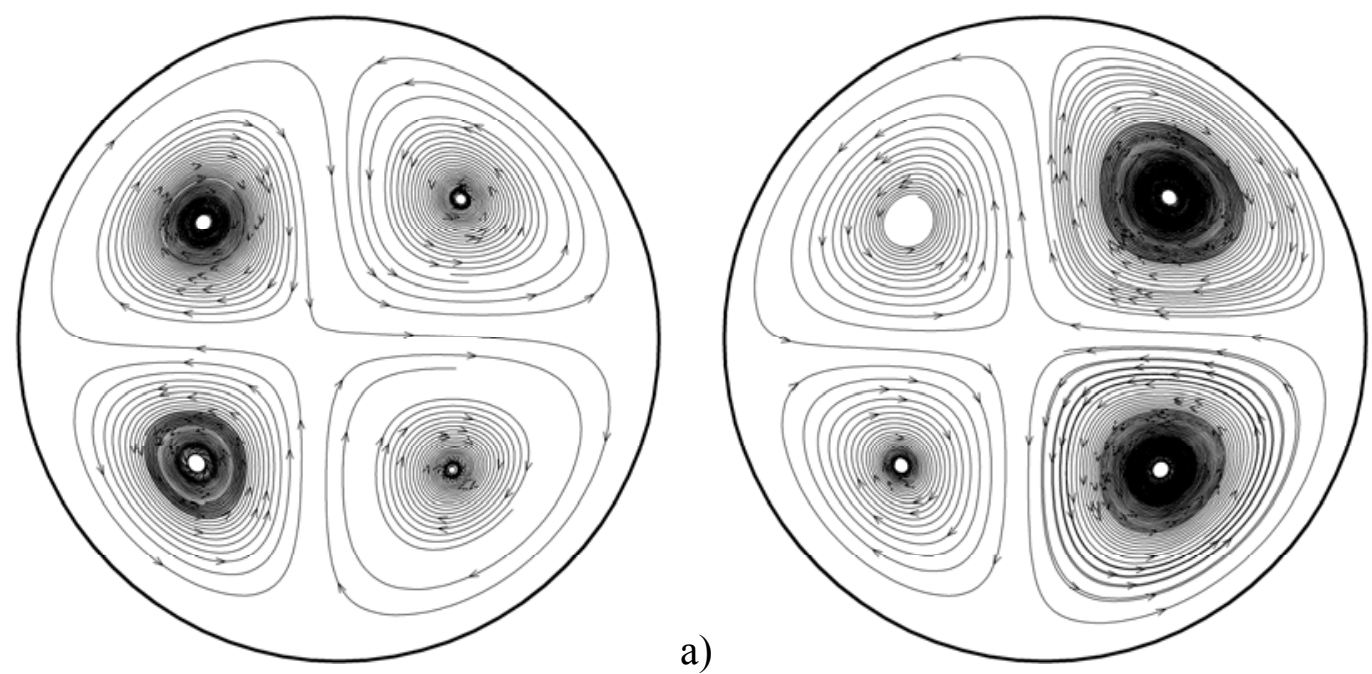

b)

Figure 15: Snapshots of velocity field in the cross section $\mathrm{z}=0$ at two instants during the period $\tau$ of oscillation (case C): a) $\left.\mathrm{t}=\mathrm{t}_{0}, \mathrm{~b}\right) \mathrm{t}=\mathrm{t}_{0}+\tau / 2(\tau=2 \pi / \varpi)$.

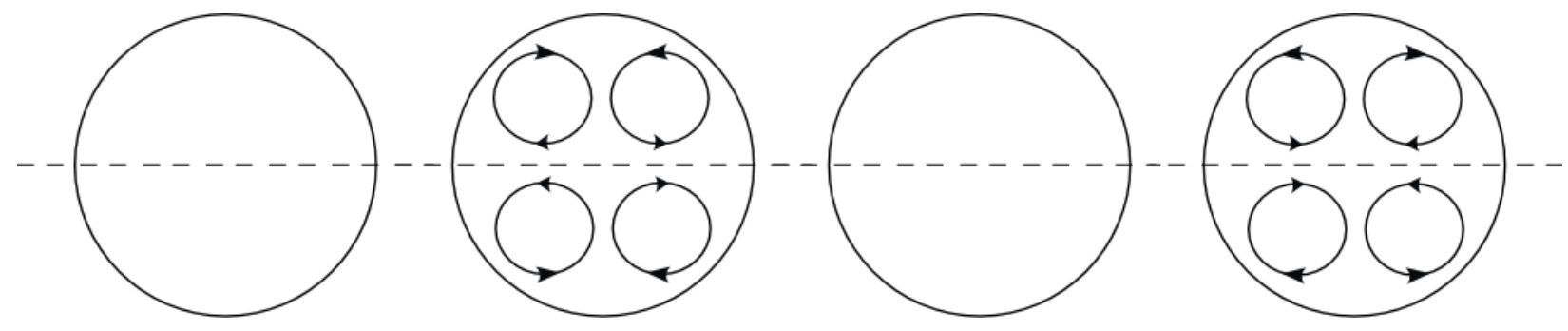

Figure 16: Sequence (sketch) showing the evolution of vibrations-induced convective cells in the generic cross section $\mathrm{z}=$ const during one period of oscillation for case $\mathrm{C}$.

A more precise idea of the threedimensional complexity of the flow in this case can be obtained by taking a look at the snapshots of azimuthal velocity isosurfaces shown in Figure 17.

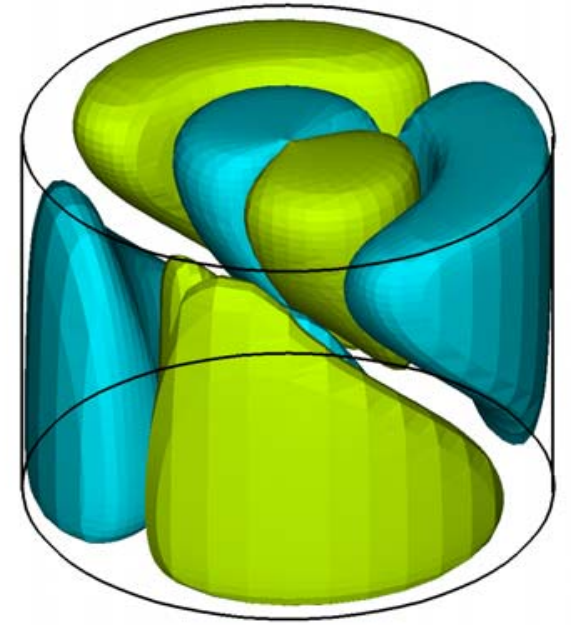

a)

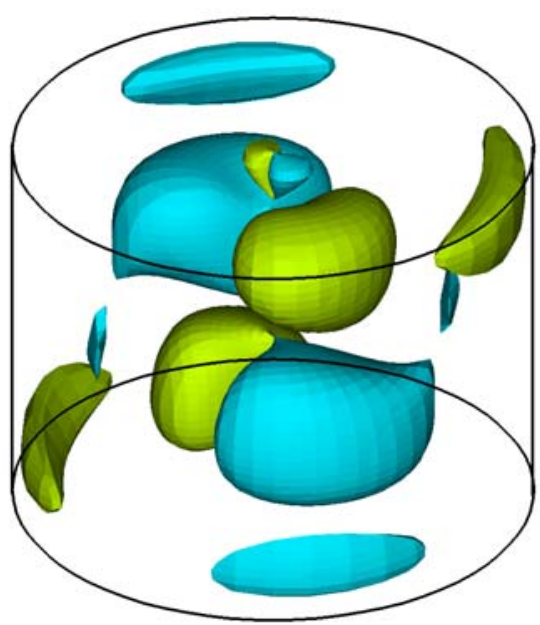

b)

Figure 17: Snapshots of 3D isosurfaces of azimuthal velocity component $w(w= \pm 1)$ for the same conditions considered in Figure 12: $\left.a) t=t_{0}, b\right) t=t_{0}+\tau / 4$. 


\section{Particle Structures}

Figure 18 illustrates that despite the increased complexity characterizing the oscillatory velocity field produced by inclined vibrations, the resulting particle accumulation structures are rather similar to those seen for the case of axial temperature gradient. Interestingly, however, as evident in Fig. 18a (which shows a lateral view of the system) the extension along the axial direction of the region affected by the presence of particles (in such a figure the white area between the external system boundary and the internal darker region indicates the zone that has been completely depleted of particles) is reduced with respect to Fig. 10. This indicates that the inclination of the imposed temperature gradient can be used to produce an axial compression of the pattern formed by the dispersed solid matter.
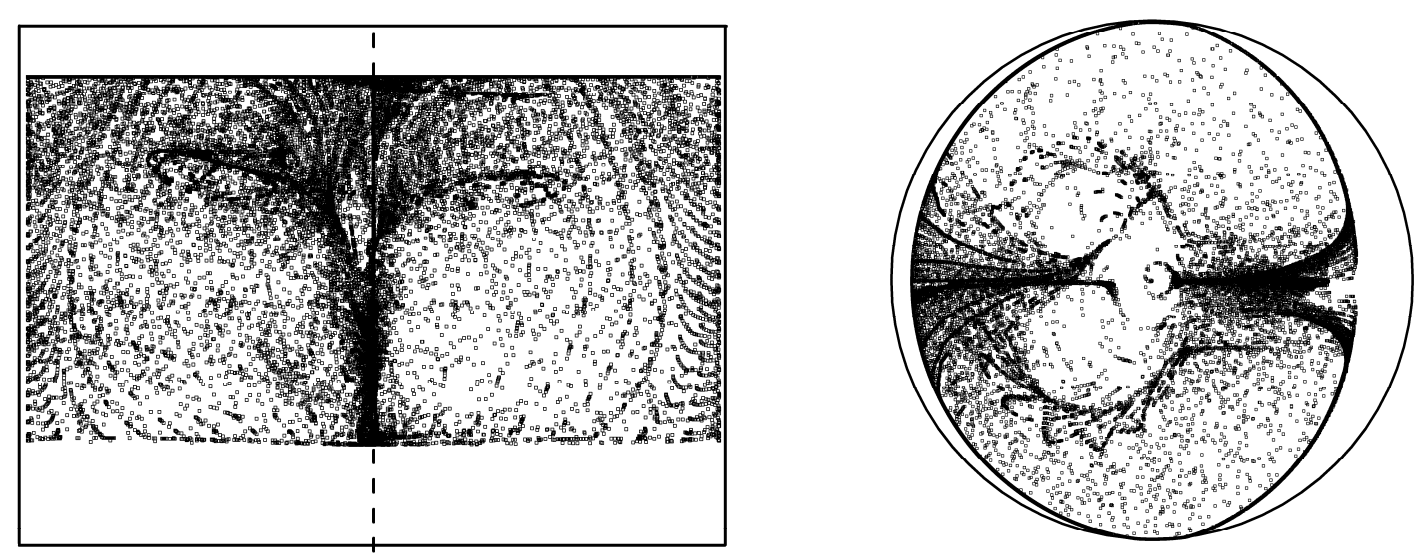

Figure 18: Snapshot of particle aggregates (lateral and top views, case C).

Moreover, accurate inspection of the lateral view reveals that some internal particle structures can be identified. Such structures, which were not present in Figure 10 and look like the curved branches of a tree, should be ascribed to the joint action exerted on the system by the axial component of imposed vibrations and the component of the imposed temperature gradient along the $\mathrm{x}$ axis (via the mechanism illustrated in Sect. IIIB2, which will tend to produce closed circuits like those seen in Fig. 13).

\section{Discussion}

As highlighted in Sect. II, hydrodynamic interactions (assumed to be absent under the assumption of a dilute solid-liquid system) play no role in the present dynamics. The cause-and-effect relationships driving particle clustering must be therefore located elsewhere.

As discussed to a certain extent in the earlier section, for the considered phenomena vibrations will be exerting their influence on particles via different separate mechanisms, one being the "direct effect" of the resulting accelerations on the particles due to their different density with respect to the surrounding fluid (which would be still present in an isothermal system) and the other being related 
to the way by which the flow of thermovibrational nature tends to transport particles according to their size and drag. The latter, however, can be further split into a "mean" effect (i.e. how the timeaveraged velocity field can influence the transports of particles) and an "instantaneous" effect related to the periodic velocity $\underline{V}^{\prime}=\underline{V}-\langle\underline{V}\rangle$ oscillating in time at the same acceleration frequency $\omega$ of the imposed inertial disturbance.

In order to discern clearly the contribution brought to the pattern by each of the above mechanisms, this section is finally devoted to an examination of the distinct behaviors which are produced when the influence of such effects is explored "separately". This approach is generally known as "modeling hierarchy" and consists of a diversity of model types in which various processes are switched on and off and the results are carefully examined.

More precisely, we compare the results of the computations obtained for the following different conditions:

a) Particles transported by the effective velocity field and subjected to the vibrational body force (full system);

b) Particles transported by the velocity field obtained subtracting the mean flow from the effective velocity distribution (particles still subjected to the vibrational body force);

c) Particles transported by the mean flow only (still subjected to the vibrational body force);

d) Particle subjected to the oscillatory body force in an isothermal system;

e) Particles transported by the mean flow only neglecting the oscillatory body force;

f) Particles transported by the effective (total) flow neglecting the oscillatory body force;

We focus again on the same case already used for code validation, namely the cylindrical enclosure with non-isothermal sidewall and adiabatic ends with vibrations along the $\mathrm{z}$ axis, for which we could measure precisely the size of the particle structures in the yz plane (Fig. 12 and Table II).

Fig. 19a and 19b immediately reveal that, apart from a very small displacement towards left of the resulting particle loops in Fig. 19b (with respect to those in Fig. 19a), subtracting the mean field to the total velocity field does not change significantly the pattern (neither form a quantitative, nor form a qualitative point of view).

Vice versa, the lack of recognizable structures in Figs. 19c clearly indicates that the oscillatory component of the velocity field $\underline{V}^{\prime}=\underline{V}-\langle\underline{V}\rangle$ is an essential ingredient without which particles do not undergo significant clustering. Indeed, although some distortion of the initially uniform distribution of particles can be seen (in agreement with the expected effect of the quadrupolar velocity field shown in Fig. 4), particles do not form dense structures. Among other things, such an argument is further supported by the results shown in Fig. 19d, where in the complete absence of convective flow, the pattern attains a trivial configuration (the particles retain a more or less even 
distribution; the compression of the pattern along the vertical direction is due to the body force that periodically displays it up and down).
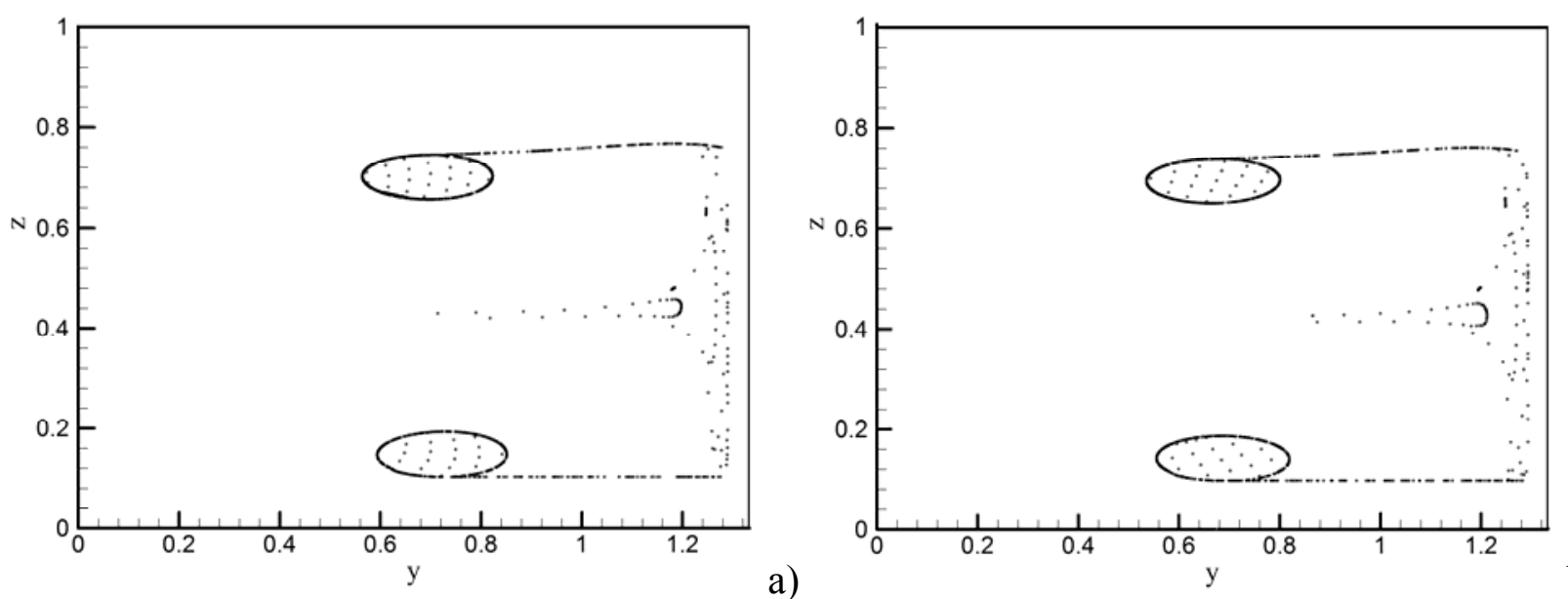

b)
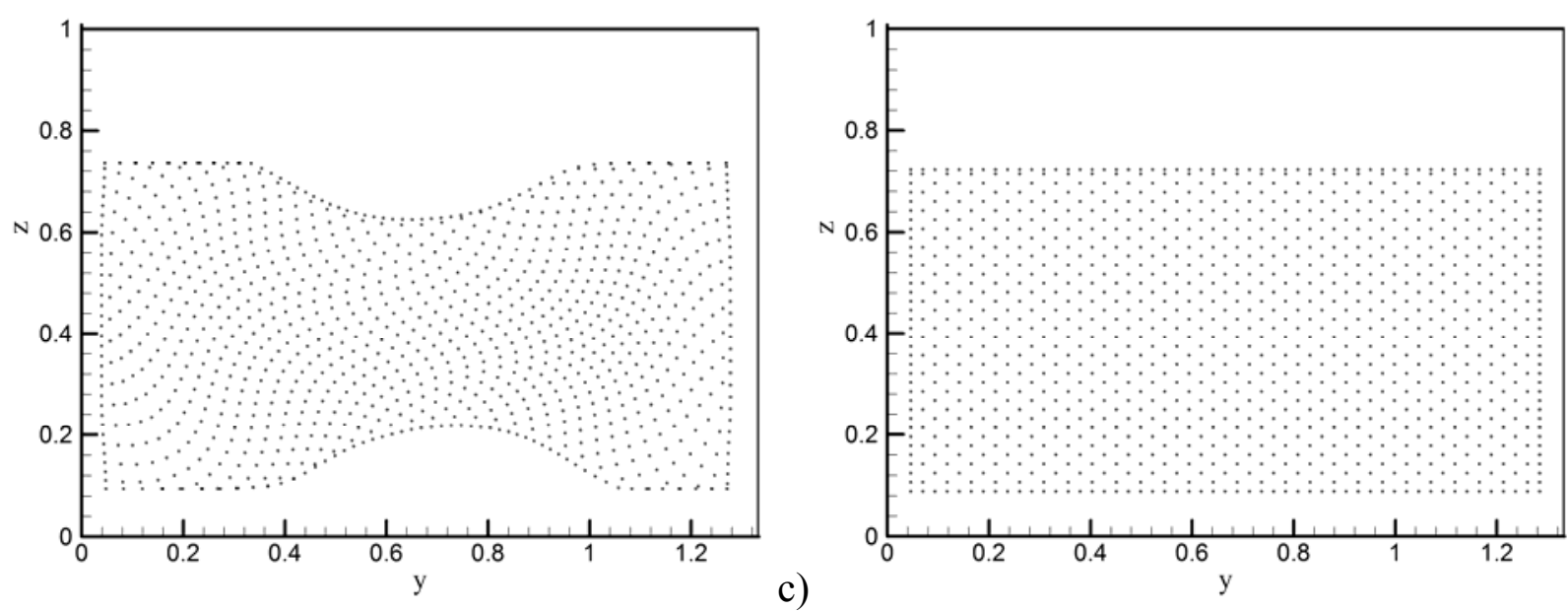

d)
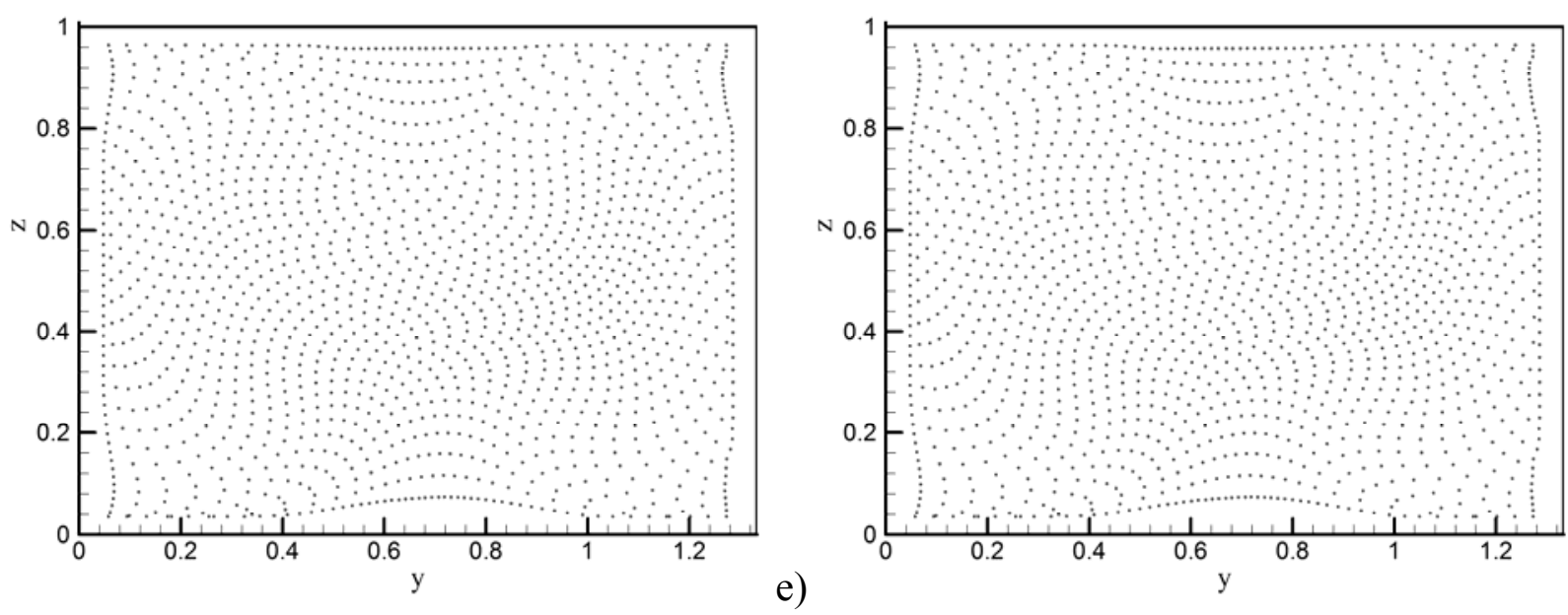

f)

Figure 19: Snapshot of particle aggregates (plane $y z)$ in the case $B\left(t=5.6 \times 10^{-1}\right)$ for different conditions: a) particles transported by the effective velocity field and subjected to the vibrational body force (full system); b) particles transported by the velocity field obtained subtracting the mean flow from the effective velocity distribution and subjected to the vibrational body force; c) particles transported by the mean flow only and subjected to the vibrational body force; $d$ ) particles subjected to vibrations in an isothermal system; e) particles transported by the mean flow only neglecting the oscillatory vibrational body force; f) particles transported by the effective (total) oscillatory flow neglecting the oscillatory vibrational body force. 
Figures $19 \mathrm{e}$ and $19 \mathrm{f}$ finally show that when the oscillatory thermovibrational body force is neglected, neither the mean flow field, nor the total field can produce particle structures (the effect of the mean velocity being limited to a weak distortion of the initial uniform particle distribution used as initial condition).

All these arguments taken together provide additional evidence to the conclusion that the coexistence of an oscillatory body force and a velocity field oscillating in time should be regarded as a necessary prerequisite for the emergence of recognizable particle agglomerates for the conditions considered here. By contrast, one may consider the role played by the time-averaged flow almost negligible in terms of particle clustering phenomena.

\section{Conclusion}

Although, in a rather unexpected way, the "multiplicity" of the resulting recognizable accumulation structures (particle-dense surfaces) is "two" for all cases considered (two more or less regular tubular structures are visible in Figs. 10, 12 and 18, regardless of whether the temperature gradient is parallel, perpendicular or inclined with respect to the symmetry axis of the cylindrical enclosure), their spatial extension (percentage of physical volume occupied), "separation" (spatial distance) change significantly. As shown by the numerical results, the intriguing diversity of particle agglomerates when the direction of the imposed temperature gradient is changed (while maintaining vibrations perpendicular to it) results from the different role/importance played by (curved or straight) boundaries in constraining particles and, from the different topology of the resulting (largescale) oscillatory thermovibrational flow (whereas the time-averaged part of the flow does not provide a substantial contribution in terms of particle clustering dynamics).

In view of the generality of the mechanisms involved, we expect the importance of this category of structure-forming processes to open the door to new strategies for the separation of phases in typical industrial applications involving cylindrical cavities or systems. There is a variety of fabrication processes (in which distinct components have to be fractionated) which may be considered as relevant examples of situations where the concepts elaborated in this work may have very interesting applications.

Although some general (qualitative) trends about the dependence of the present phenomena on the vibration frequency and amplitude (which in the present study were fixed) may be elaborated "a priori" on the basis of earlier analyses dealing with the cubic cavity [12], future work shall be devoted to parametric quantitative evaluation of the influence of the problem non-dimensional parameters on the formation time and the size and position in space of the new particle structures revealed here. 


\section{References}

[1] Segurado J., González C., LLorca J., (2003), A numerical investigation of the effect of particle clustering on the mechanical properties of composites, Acta Materialia 51, 2355-2369.

[2] Hassan S., Lyubimova T. P., Lyubimov D. V., Kawaji M., (2006), Effects of vibrations on particle motion near a wall: Existence of attraction force, Int. J. Multiphase Flow, 32 (9), 1037-1054. [3] Lappa M., (2016), On the nature, formation and diversity of particulate coherent structures in Microgravity Conditions and their relevance to materials science and problems of Astrophysical interest, Geophysical and Astrophysical Fluid Dynamics, 110(4): 348-386.

[4] Melnikov D., Pushkin D., and Shevtsova V., (2011) Accumulation of particles in timedependent thermocapillary flow in a liquid bridge. Modeling of experiments, Eur. Phys. J. Special Topics, 192, 29-39.

[5] Melnikov D.E., Pushkin D.O., Shevtsova V.M., (2013), Synchronization of finite-size particles by a traveling wave in a cylindrical flow, Phys Fluids, 25 (9), 092108

[6] Ueno I., Abe Y., Noguchi K., and Kawamura H., (2008), Dynamic particle accumulation structure (PAS) in half-zone liquid bridge - Reconstruction of particle motion by 3-D PTV, Adv. Space Res., 41, 2145.

[7] Gotoda M., Sano T., Kaneko T., and Ueno I., (2015), Evaluation of existence region and formation time of particle accumulation structure (PAS) in half-zone liquid bridge, Eur. Phys. J. Special Topics, 224, 299.

[8] Pushkin D., Melnikov D., Shevtsova V., (2011), Ordering of Small Particles in OneDimensional Coherent Structures by Time-Periodic Flows, Phys. Rev. Lett., 106, 234501.

[9] Schwabe D., Tanaka S., Mizev A. and Kawamura H., (2006), Particle accumulation structures in time-dependent thermocapillary flow in a liquid bridge under microgravity, Microgravity Sci. Technol. 18, 117.

[10] Schwabe D. and Mizev A.I., (2011), Particles of different density in thermocapillary liquid bridges under the action of travelling and standing hydrothermal waves, Eur. Phys. J. Special Topics, 192, 13-27

[11] Kozlov V.G., Ivanova A.A. and Evesque P., (2006), Block stratification of sedimenting granular matter in a vessel due to vertical vibration, Fluid Dyn. Mater. Process., 2(3), 203-210.

[12] Lappa M., (2014), The patterning behaviour and accumulation of spherical particles in a vibrated non-isothermal liquid, Phys. Fluids, 26(9), 093301 (22 pages).

[13] Crespo Del Arco E. and Bontoux P., (1989), Numerical simulations and analysis of axisymmetric convection in a vertical cylinder: An effect of Prandtl number, Phys Fluids, A1: 1348-1359.

[14] Crespo del Arco E., Bountoux P., Sani R. L., Hardin G., Extrémet G. P., (1988), Steady and oscillatory convection in vertical cylinders heated from below. Numerical simulation of asymmetric flow regimes, Adv. Space Res., 8(12): 281-292

[15] Smutek C., Bontoux P., Roux B., Schiroky G.H., Hurford A.C., (1985), Three-dimensional convection in horizontal cylinders - Numerical solutions and comparison with experimental and analytical results, Numer. Heat Transfer, Part A: Applications, 8(5): 613-631.

[16] Bontoux P., Roux B., Schiroky G. H., Markham B. L., Rosenberger F., (1986), Convection in the vertical midplane of a horizontal cylinder. Comparison of two-dimensional approximations with three-dimensional results, Int. J. Heat Mass Transfer, 29 (2): 227-240 
[17] Bontoux P., Smutek C., Roux B. and Lacroix J.M., (1986), Three-dimensional buoyancydriven flows in cylindrical cavities with differentially heated endwalls. Part 1 . Horizontal cylinders, J. Fluid Mech., 169: 211-227.

[18] Vaux S., BenHadid H. and Henry D., (2006), Study of the hydrodynamic instabilities in a differentially heated horizontal circular cylinder corresponding to a Bridgman growth configuration, J. Cryst.Growth, 290: 674-682.

[19] Brooks I.H. and Ostrach S., (1970), An experimental investigation of natural convection in a horizontal cylinder, J. Fluid Mech., 44: 545-561.

[20] Ostrach S. and Hantman R.G., (1981), Natural convection inside a horizontal cylinder, Chem. Engr. Commun., 9(1): 213-243.

[21] Xin S., Le Quéré P. and Daube O., (1997), Natural convection in a differentially heated horizontal cylinder : Effects of Prandtl number on flow structure and instability, Phys. Fluids, 9(4): 1014-1033.

[22] Gelfgat A.Yu., Bar-Yoseph P.Z., Solan A., (2000), Axisymmetry breaking instabilities of Natural convection in a vertical Bridgman growth configuration, J. Cryst. Growth, 220: 316-325.

[23] Alexander J.I.D., Ouazzani J., and Rosenberger F., (1991), Analysis of the low gravity tolerance of Bridgman-Stockbarger crystal growth, II. Transient and periodic accelerations, J. Cryst. Growth, 113(1-2): 21-38.

[24] Alexander J.I.D., Garandet J.-P., Favier J.J., and Lizee A., (1997), g-Jitter effects on segregation during directional solidification of tin-bismuth in the MEPHISTO furnace facility, J.Cryst. Growth, 178: 657-661.

[25] Wadih M. and Roux B., (1988), Natural convection in a long vertical cylinder under gravity modulation, J. Fluid Mech., 193: 391-415.

[26] Zharikov E.V., Prihodko L.V., Storozhev N.R., (1990), Fluid flow formation resulting from forced vibration of a growing crystal, J. Cryst. Growth, 99: 910-914.

[27] Wheeler A.A., McFadden G.B., Murray B.T., Coriell S.R., (1991), Convective stability in the Rayleigh-Bénard and directional solidification problems: high-frequency gravity modulation, Phys. Fluids A, 3: 2847-2858.

[28] Murray B.T., Coriell S.R., McFadden G.B., (1992), The effect of gravitational modulations on convection in vertical bridgman growth, Proceedings VIIIth European Symposium on Materials and Fluid Sciences in Microgravity, Brussels, Belgium 12-16 April , 1992, ESA SP333: 503-506.

[29] Murray B.T., Coriell S.R., McFadden G.B., Wheeler A.A. and Saunders B.V., (1993), Gravitational modulation of thermosolutal convection during directional solidification, J. Cryst. Growth, 129: 70-80.

[30] Ivanova A.A. and Kozlov V.G., (2003), Thermal Vibrational Convection in a Cavity under Nontranslational Oscillations, Fluid Dynamics, 38(3), 372-386.

[31] Kozlov, V.G. and Selin, N.V. (2006), Pendulum thermal vibrational convection in a liquid layer with internal heat generation, Fluid Dyn. Mater. Process., 2, No. 2, 107-117

[32] Kuhlmann H.C., Lappa M., Melnikov D., Mukin R., Muldoon F.H., Pushkin D., Shevtsova V.S., and Ueno I., (2014), The JEREMI-Project on thermocapillary convection in liquid bridges. Part A: Overview of Particle Accumulation Structures, Fluid Dyn. Mater. Process., 10 (1), 1-26.

[33] Lappa M., (2013), Assessment of the role of axial vorticity in the formation of Particle Accumulation Structures in supercritical Marangoni and hybrid thermocapillary-rotation-driven flows, Phys. Fluids, 25(1), 012101. 
[34] Lappa M., (2014), Stationary Solid Particle Attractors in Standing Waves, Phys. Fluids, 26(1), 013305 (12 pages).

[35] Lappa M., (2013), On the Existence and Multiplicity of One-dimensional Solid Particle Attractors in Time-dependent Rayleigh-Bénard Convection, Chaos, 23(1), 013105.

[36] Monti R., Savino R., Lappa M., (2001), On the convective disturbances induced by g-jitter on the space station, Acta Astronautica, 48 (5-12), 603-615.

[37] Lappa M. and Carotenuto L., (2003), Effect of convective disturbances induced by g-jitter on the periodic precipitation of lysozyme, Microgravity Sci. Tech., XIV/2, 41-56.

[38] Savino R. and Lappa M., (2003), Assessment of the thermovibrational theory: application to gjitter on the Space-station, Journal of Spacecraft and Rockets, 40 (2), 201-210.

[39] Mialdun A., Ryzhkov I.I., Melnikov D.E., and Shevtsova V., (2008), Experimental Evidence of Thermal Vibrational Convection in a Nonuniformly Heated Fluid in a Reduced Gravity Environment, Phys. Rev. Lett., 101, 084501.

[40] Shevtsova V., Mialdun A., Melnikov D., Ryzhkov I., Gaponenko Y., Saghir Z., Lyubimova T., and Legros J. C., (2011b), The IVIDIL experiment onboard the ISS: Thermodiffusion in the presence of controlled vibrations, Compt. Rend. Mécaniq., 339, No. (5), 310-317.

[41] Lappa M., (2013), On the variety of particle accumulation structures under the effect of gjitters, J. Fluid Mech., 726, 160-195.

[42] Lappa M., (2016), Control of convection patterning and intensity in shallow cavities by harmonic vibrations, Microgravity Science \& Technology, 28(1), 29-39.

[43] Maxey M. R. and Riley J. J., (1983), Equation of motion for a small rigid sphere in a nonuniform flow, Phys. Fluids, 26, 883-889.

[44] Balboa Usabiaga F., Pagonabarraga I., and Delgado-Buscalioni R., (2013), Inertial coupling for point particle fluctuating hydrodynamics, J. Comp. Phys., 235, 701-722.

[45] Esmaeeli A., (2005), Phase Distribution of Bubbly Flows under Terrestrial and Microgravity Conditions, Fluid Dyn. Mater. Process., 1, No. 1, 63-80

[46] Derksen J.J. and Eskin D., (2011), Flow-Induced Forces in Agglomerates, Fluid Dyn. Mater. Process., 7(4), 341-356.

[47] Langbein D., (1991), Motion of Ensembles of Spherical Particles in a Fluid Due to g-jitter, Adv. Space Res., 11(7), 189-196.

[48] Hassan S. and Kawaji M., (2008), The effects of vibrations on particle motion in a viscous fluid cell, J. Appl. Mech. 75, 031012.

[49] Fletcher C.A.J., (1991), Computational techniques for fluid-dynamics, (Springer Verlag, Berlin, 1991).

[50] Khallouf H., Gershuni G. Z. and Mojtabi A., (1995), Numerical study of two-dimensional thermo-vibrational convection in rectangular cavities, Num. Heat Trans. A, 27, 297-305.

[51] Mialdun A., Ryzhkov I.I., Melnikov D.E., and Shevtsova V., (2008), Experimental Evidence of Thermal Vibrational Convection in a Nonuniformly Heated Fluid in a Reduced Gravity Environment, Phys. Rev. Lett., 101, 084501. 